UNIVERSITÉ CLERMONT AUVERGNE

UFR DE MÉDECINE ET DES PROFESSIONS PARAMÉDICALES

THÈSE D'EXERCICE

pour le

DIPLÔME D’ÉTAT DE DOCTEUR EN MÉDECINE

par

CAMBOU Ludivine

Présentée et soutenue publiquement le 9 Avril 2021

\title{
THROMBOSE VEINEUSE PRÉCOCE DU GREFFON RÉNAL : QUELLE PRISE EN CHARGE?
}

\section{Président du Jury:}

Monsieur GUY Laurent, Professeur, Faculté de Médecine de Clermont-Ferrand

Membres du Jury:

Madame HENG Anne-Elisabeth, Professeur, Faculté de Médecine de Clermont-Ferrand Monsieur SCHMIDT Jeannot, Professeur, Faculté de Médecine de Clermont-Ferrand Monsieur GARROUSTE Cyril, Docteur, CHU Gabriel Montpied, Clermont-Ferrand Madame MILLET Clémentine, Docteur, CHU Gabriel Montpied, Clermont-Ferrand 

UNIVERSITÉ CLERMONT AUVERGNE

UFR DE MÉDECINE ET DES PROFESSIONS PARAMÉDICALES

THÈSE D'EXERCICE

pour le

DIPLÔME D’ÉTAT DE DOCTEUR EN MÉDECINE

par

CAMBOU Ludivine

Présentée et soutenue publiquement le 9 Avril 2021

\section{THROMBOSE VEINEUSE PRÉCOCE DU GREFFON RÉNAL : QUELLE PRISE EN CHARGE?}

Président du Jury :

Monsieur GUY Laurent, Professeur, Faculté de Médecine de Clermont-Ferrand

Membres du Jury :

Madame HENG Anne-Elisabeth, Professeur, Faculté de Médecine de Clermont-Ferrand Monsieur SCHMIDT Jeannot, Professeur, Faculté de Médecine de Clermont-Ferrand Monsieur GARROUSTE Cyril, Docteur, CHU Gabriel Montpied, Clermont-Ferrand Madame MILLET Clémentine, Docteur, CHU Gabriel Montpied, Clermont-Ferrand 


\section{UNIVERSITE CLERMONT AUVERGNE}

PRESIDENTS HONORAIRES

UNIVERSITE D'AUVERGNE

PRESIDENTS HONORAIRES

UNIVERSITE BLAISE PASCAL
: JOYON Louis

: DOLY Michel

: TURPIN Dominique

: VEYRE Annie

: DULBECCO Philippe

: ESCHALIER Alain

: CABANES Pierre

: FONTAINE Jacques

: BOUTIN Christian

: MONTEIL Jean-Marc

: ODOUARD Albert

: LAVIGNOTTE Nadine

: BERNARD Mathias : DEQUIEDT Vianney : WILLIAMS Benjamin : HENRARD Pierre VICE

: PEYRARD Françoise : PAQUIS François

FORMATION ET DE LA VIE UNIVERSITAIRE

DIRECTEUR GENERAL DES SERVICES

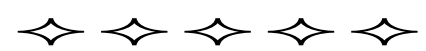

\section{UFR DE MEDECINE \\ ET DES PROFESSIONS \\ PARAMEDICALES}

DOYENS HONORAIRES

: DETEIX Patrice

: CHAZAL Jean

DOYEN

RESPONSABLE ADMINISTRATIVE

: CLAVELOU Pierre

: ROBERT Gaëlle 


\section{LISTE DU PERSONNEL ENSEIGNANT}

\section{PROFESSEURS HONORAIRES :}

MM. BACIN Franck - BEGUE René-Jean - BOUCHER Daniel - BOURGES Michel BUSSIERE Jean-Louis - CANO Noël - CASSAGNES Jean - CATILINA Pierre CHABANNES Jacques - CHAZAL Jean - CHIPPONI Jacques - CHOLLET Philippe COUDERT Jean - DASTUGUE Bernard - DAUPLAT Jacques - DECHELOTTE Pierre - DEMEOCQ François - DE RIBEROLLES Charles - ESCANDE Georges Mme FONCK Yvette - MM. GENTOU Claude - GLANDDIER Gérard - Mme GLANDDIER Phyllis - M. JACQUETIN Bernard - Mme LAVARENNE Jeanine MM. LAVERAN Henri - LESOURD Bruno - LEVAI Jean-Paul - MAGE Gérard MALPUECH Georges - MARCHEIX Jean-Claude - MICHEL Jean-Luc - MOLINA Claude - MONDIE Jean-Michel - PERI Georges - PETIT Georges - PHILIPPE Pierre PLAGNE Robert - PLANCHE Roger - PONSONNAILLE Jean - REY Michel - Mme RIGAL Danièle - MM. ROZAN Raymond - SCHOEFFLER Pierre - SIROT Jacques SOUTEYRAND Pierre - TANGUY Alain - TERVER Sylvain - THIEBLOT Philippe TOURNILHAC Michel - VANNEUVILLE Guy - VIALLET Jean-François - Mle VEYRE Annie

\section{PROFESSEURS EMERITES :}

MM. - BEYTOUT Jean - BOITEUX Jean-Paul - BOMMELAER Gilles - CHAMOUX Alain - DETEIX Patrice - DUBRAY Claude - ESCHALIER Alain - IRTHUM Bernard - KEMENY Jean- Louis - LABBE André - Mme LAFEUILLE Hélène - MM. LEMERY Didier LUSSON Jean- René - RIBAL Jean-Pierre

\section{PROFESSEURS DES UNIVERSITES-PRATICIENS HOSPITALIERS}

\section{PROFESSEURS DE}

CLASSE EXCEPTIONNELLE

M. VAGO Philippe

M. AUMAITRE Olivier

M. LABBE André

M. AVAN Paul

M. DURIF Franck

M. BOIRE Jean-Yves

M. BOYER Louis

M. POULY Jean-Luc

M. CANIS Michel

Mme PENAULT-LLORCA Frédérique

M. BAZIN Jean-Etienne
Histologie-Embryologie Cytogénétique

Médecine Interne

Pédiatrie

Biophysique et Traitement de l'Image

Neurologie

Biostatistiques, Informatique

Médicale et Technologies de

Communication

Radiologie et Imagerie

Médicale option

Clinique

Gynécologie et Obstétrique

Gynécologie-Obstétrique

Anatomie et Cytologie Pathologiques

Anesthésiologie et 
M. BIGNON Yves Jean

M. BOIRIE Yves

M. CLAVELOU Pierre

M. DUBRAY Claude

M. GILAIN Laurent

M. LEMAIRE Jean-Jacques

M. CAMILLERI Lionel

Vasculaire

M. DAPOIGNY Michel

M. LLORCA Pierre-Michel

M. PEZET Denis

M. SOUWEINE Bertrand

M. BOISGARD Stéphane

Traumatologie

Mme DUCLOS Martine

M. SCHMIDT Jeannot

M. BERGER Marc

M. GARCIER Jean-Marc

Médicale

M. ROSSET Eugénio

M. SOUBRIER Martin
Réanimation

Chirurgicale

Cancérologie option Biologique

Nutrition Humaine

Neurologie

Pharmacologie Clinique

O.R.L.

Neurochirurgie

Chirurgie Thoracique et Cardio-

Gastro-Entérologie

Psychiatrie d'Adultes

Chirurgie Digestive

Réanimation Médicale

Chirurgie Orthopédique et

Physiologie

Thérapeutique

Hématologie

Anatomie-Radiologie et Imagerie

Chirurgie Vasculaire

Rhumatologie

\section{PROFESSEURS DE}

lère CLASSE

\begin{abstract}
M. CAILLAUD Denis
M. VERRELLE Pierre

M. CITRON Bernard

M. D'INCAN Michel
\end{abstract}

Mme JALENQUES Isabelle

Mle BARTHELEMY Isabelle

M. GERBAUD Laurent

M. TAUVERON Igor

Métaboliques

M. MOM Thierry

M. RICHARD Ruddy

M. RUIVARD Marc

M. SAPIN Vincent

M. BAY Jacques-Olivier

M. COUDEYRE Emmanuel

Mme GODFRAIND Catherine

M. ABERGEL Armando

M. LAURICHESSE Henri

M. TOURNILHAC Olivier

M. CHIAMBARETTA Frédéric

M. FILAIRE Marc

M. GALLOT Denis
Pneumo-phtisiologie

Radiothérapie option Clinique

Cardiologie et Maladies Vasculaires

Dermatologie -Vénéréologie

Psychiatrie d'Adultes

Chirurgie Maxillo-Faciale

Epidémiologie, Economie de

la Santé et Prévention

Endocrinologie et Maladies

Oto-Rhino-Laryngologie

Physiologie

Médecine Interne

Biochimie et Biologie Moléculaire

Cancérologie

Médecine Physique et de Réadaptation

Anatomie et Cytologie Pathologiques

Hépatologie

Maladies Infectieuses et Tropicales

Hématologie

Ophtalmologie

Anatomie - Chirurgie

Thoracique et Cardio-

Vasculaire

Gynécologie-Obstétrique 
M. GUY Laurent

M. TRAORE Ousmane

M. ANDRE Marc

M. BONNET Richard

M. CACHIN Florent

M. COSTES Frédéric

M. FUTIER Emmanuel

Mme HENG Anne-Elisabeth

M. MOTREFF Pascal

Mme PICKERING Gisèle

M. RABISCHONG Benoît
Urologie

Hygiène Hospitalière

Médecine Interne

Bactériologie, Virologie

Biophysique et Médecine Nucléaire

Physiologie

Anesthésiologie-Réanimation

Néphrologie

Cardiologie

Pharmacologie Clinique

Gynécologie Obstétrique

PROFESSEURS DE 2ème CLASSE

Mme CREVEAUX Isabelle

M. FAICT Thierry

la Santé

Mme KANOLD LASTAWIECKA Justyna

M. TCHIRKOV Andréï

M. CORNELIS François

M. DESCAMPS Stéphane

Traumatologique

M. POMEL Christophe

M. CANAVESE Fédérico

M. LESENS Olivier

M. AUTHIER Nicolas

M. BROUSSE Georges

M. BUC Emmanuel

M. CHABROT Pascal

M. LAUTRETTE Alexandre

M. AZARNOUSH Kasra

cardiovasculaire

Mme BRUGNON Florence

Mme HENQUELL Cécile

M. ESCHALIER Romain

M. MERLIN Etienne

Mme TOURNADRE Anne

M. DURANDO Xavier

M. DUTHEIL Frédéric

Mme FANTINI Maria Livia

M. SAKKA Laurent

M. BOURDEL Nicolas

M. GUIEZE Romain

M. POINCLOUX Laurent

M. SOUTEYRAND Géraud

M. EVRARD Bertrand

M. POIRIER Philippe
Biochimie et Biologie Moléculaire Médecine Légale et Droit de

Pédiatrie

Cytologie et Histologie

Génétique

Chirurgie Orthopédique et

Cancérologie - Chirurgie Générale

Chirurgie Infantile

Maladies Infectieuses et Tropicales

Pharmacologie Médicale

Psychiatrie Adultes/Addictologie

Chirurgie Digestive

Radiologie et Imagerie Médicale

Néphrologie Réanimation Médicale

Chirurgie Thoracique et

Biologie et Médecine du

Développement et de la

Reproduction

Bactériologie Virologie

Cardiologie

Pédiatrie

Rhumatologie

Cancérologie

Médecine et Santé au Travail

Neurologie

Anatomie - Neurochirurgie

Gynécologie-Obstétrique

Hématologie

Gastroentérologie

Cardiologie

Immunologie

Parasitologie et Mycologie 


\section{PROFESSEURS DES UNIVERSITES}

M. CLEMENT Gilles Médecine

Générale Mme MALPUECH-BRUGERE Corinne Nutrition

Humaine

M. VORILHON Philippe Médecine Générale

\section{PROFESSEURS ASSOCIES DES UNIVERSITES}

Mme BOTTET-MAULOUBIER Anne

M. CAMBON Benoît

M. TANGUY Gilles
Médecine Générale

Médecine Générale

Médecine Générale

\section{MAITRES DE CONFERENCES DES UNIVERSITES - PRATICIENS HOSPITALIERS}

\section{MAITRES DE CONFERENCES} HORS CLASSE

Mme CHAMBON Martine Mme BOUTELOUP Corinne
Bactériologie Virologie

Nutrition
M. MORVAN Daniel

Mle GOUMY Carole

Mme FOGLI Anne

Mle GOUAS Laetitia

M. MARCEAU Geoffroy

Mme MINET-QUINARD Régine

M. ROBIN Frédéric

Mle VERONESE Lauren

M. DELMAS Julien

Mle MIRAND Audrey

M. OUCHCHANE Lemlih

M. LIBERT Frédéric

Mle COSTE Karen
Biophysique et Traitement de l'Image

Cytologie et Histologie, Cytogénétique

Biochimie Biologie Moléculaire

Cytologie et Histologie, Cytogénétique

Biochimie Biologie Moléculaire

Biochimie Biologie Moléculaire

Bactériologie

Cytologie et Histologie, Cytogénétique

Bactériologie

Bactériologie Virologie

Biostatistiques, Informatique

Médicale et Technologies de

Communication

Pharmacologie Médicale

Pédiatrie 
Mle AUMERAN Claire

Mme CASSAGNES Lucie

M. LEBRETON Aurélien

M. BUISSON Anthony
Hygiène Hospitalière

Radiologie et Imagerie Médicale

Hématologie

Gastroentérologie

\section{MAITRES DE CONFERENCES DE}

2ème CLASSE

Mme PONS Hanaë

M. JABAUDON-GANDET Matthieu

Chirurgicale

M. BOUVIER Damien

M. COLL Guillaume

Mme SARRET Catherine

M. MAQDASY Salwan

Mme NOURRISSON Céline
Biologie et Médecine du

Développement et de la Reproduction

Anesthésiologie - Réanimation

Biochimie et Biologie Moléculaire

Neurochirurgie

Pédiatrie

Endocrinologie, Diabète et

Maladies Métaboliques

Parasitologie - Mycologie

\section{MAITRES DE CONFERENCES DES UNIVERSITES}

Mme VAURS-BARRIERE Catherine

M. BAILLY Jean-Luc

Mle AUBEL Corinne

M. BLANCHON LoÏc

Mle GUILLET Christelle

M. BIDET Yannick

M. MARCHAND Fabien

M. DALMASSO Guillaume

M. SOLER Cédric

M. GIRAUDET Fabrice

l'Image Mme VAILLANT-ROUSSEL Hélène

Mme LAPORTE Catherine

M. LOLIGNIER Stéphane

Mme MARTEIL Gaëlle

M. PINEL Alexandre

M. PIZON Frank
Biochimie Biologie Moléculaire

Bactériologie Virologie

Oncologie Moléculaire

Biochimie Biologie Moléculaire

Nutrition Humaine

Oncogénétique

Pharmacologie Médicale

Bactériologie

Biochimie Biologie Moléculaire

Biophysique et Traitement de

Médecine Générale

Médecine Générale

Neurosciences - Neuropharmacologie

Biologie de la Reproduction

Nutrition Humaine

Santé Publique

\section{MAITRES DE CONFERENCES ASSOCIES DES UNIVERSITES}

M. BERNARD Pierre

Mme ESCHALIER Bénédicte

Mme RICHARD Amélie

M. TESSIERES Frédéric
Médecine Générale

Médecine Générale

Médecine Générale

Médecine Générale 
Àmon père, 


\section{REMERCIEMENTS}

À NOTRE PRESIDENT DU JURY

\section{Monsieur le Professeur Laurent GUY,}

Merci de votre confiance et de me faire l'honneur de présider le jury de ma thèse.

Initialement orientée vers une autre spécialité chirurgicale, vous m'avez acceptée dans votre équipe, et je vous en serai toujours reconnaissante.

Dans le service comme au bloc opératoire, votre regard paternaliste et votre encadrement nous ont permis de progresser. L'exigence que vous vous imposez et votre rigueur professionnelle, sont un exemple pour nous tous, et j'espère pouvoir être à la hauteur de votre enseignement.

Soyez assuré de ma reconnaissance et de mon plus grand respect. 


\section{À NOTRE JURY DE THESE}

\section{Madame le Professeur Anne-Elisabeth HENG,}

Vous me faites l'honneur de siéger dans mon jury de thèse.

Merci de bien vouloir partager votre riche expérience et votre regard expert sur la transplantation rénale, en jugeant mon travail.

J'ai beaucoup apprécié de travailler en collaboration avec vous et toute votre équipe, tout au long de mon internat et j'espère pouvoir continuer à le faire.

\section{Monsieur le Professeur Jeannot SCHMIDT,}

Merci de me faire l'honneur de participer à mon jury de thèse et d'avoir accepté d'évaluer mon travail.

Compte tenu de vos compétences reconnues dans votre domaine, et particulièrement dans la prise en charge des thromboses veineuses, je ne pouvais pas espérer mieux pour juger mon travail.

\section{Monsieur le Docteur Cyril Garrouste}

Je tiens à te remercier de faire partie de mon jury de thèse.

Merci pour ton soutien et ta grande implication dans mon travail. Ton aide a été précieuse, tout au long de ce projet, et je t'en suis vraiment reconnaissante.

La collaboration entre urologues et néphrologues a été très enrichissante, et je souhaite que nos résultats soient utiles à nos deux spécialités.

C'était vraiment agréable de travailler avec toi et j'espère que j'aurai le plaisir de renouveler l'expérience. 


\section{À NOTRE DIRECTRICE DE THÈSE}

\section{Madame le Docteur Clémentine MILLET,}

Merci d'avoir accepté d'être ma directrice de thèse et de m'avoir guidée tout au long de ce travail. Ton aide et tes conseils avisés ont été indispensables ces dernières semaines.

J'ai beaucoup appris à tes côtés. Je respecte ta qualité et ta rigueur chirurgicale, ta conscience professionnelle, ton engagement auprès des patients, et ton implication dans notre formation avec bonne humeur et intégrité. Tes remarques peuvent être parfois pimentées, mais l'objectiffinal est toujours de nous faire progresser.

J'espère pouvoir retranscrire dans ma pratique future, ce que tu nous as transmis.

Je te remercie aussi pour ton soutien, pour ta confiance, pour ta bienveillance et évidemment pour tous les fous rires qu'on a partagés durant ces cinq années. J'ai de la chance de pouvoir te compter parmi mes collègues, mais surtout parmi mes amis.

Je suis ravie de t'avoir eue comme directrice de thèse, et j'espère que tu continueras d'encadrer d'autres thèses dans le futur. 


\section{À MA FAMILLE}

\section{À mon Père,}

Avec ce travail, je termine mes études, et j'aurais tellement aimé que tu sois à mes côtés pour voir que j'y suis enfin arrivée, car c'est en grande partie grâce à toi. Tu t'es battu toute ta vie pour nous donner un avenir, mais la vie a été trop injuste pour te laisser en profiter. Merci d'avoir toujours cru en moi, de m'avoir soutenue quand même moi je baissais les bras, de m'avoir aidé à me relever à chaque fois que je tombais. Tu as été un père exemplaire. Tu nous disais tout le temps que l'important c'était de faire de son mieux, et c'est ce que j'ai essayé de faire. J'espère vraiment que ce travail t'aurait rendu fier Papa. Je n'oublierai jamais qui je suis et d'où je viens, et j'essaierai d'appliquer dans ma vie toutes les belles valeurs que tu as voulu nous transmettre.

\section{À ma mère,}

C'est aussi grâce à toi maman que j'y suis arrivée. Pendant toutes ces longues années, tu m'as toujours épaulée, et tu as toujours été là pour moi. Toi aussi tu t'es acharnée au travail pour nous donner une chance de réussir. Tu nous as souvent répétés que l'important dans la vie c'était de faire quelque chose qui nous plaisait. Grâce à papa et toi, c'est chose faite. Merci pour ton réconfort, pour ta générosité et pour ces milliers de plats délicieux que tu m'as cuisiné pendant toutes mes études. Merci aussi pour ton dévouement, et pour ta patience toutes les fois où $\mathrm{j}$ 'ai su pu être insupportable. Je suis très fière de t'avoir comme mère, et j'espère qu'à ton tour tu seras fière de moi.

\section{À Cédric,}

Mille mercis mon cœur pour tout ce que tu as fait pour moi, merci d'avoir été à mes côtés toutes ces années. Tu m'as soutenu dans les meilleurs moments comme dans les pires. Ça n'a pas toujours été facile, mais on a fait face ensemble. Sans toi, je n'en serais sûrement pas là aujourd'hui. J'ai de la chance de t'avoir dans ma vie. Merci de continuer à me supporter, parce qu'au fond je sais bien que je ne suis pas très facile à vivre. Merci d'être toi, et de me rappeler, quand je me perds, les vraies priorités de la vie. Je t'aime et je suis très heureuse de t'avoir près de moi.

\section{À mes sours, Vanessa et Maéva,}

J'ai tellement de chance de vous avoir comme sœurs. Vous avez été d'un soutien sans faille depuis le début et je vous en suis vraiment très reconnaissante. Merci Vaness pour toutes les fois où tu m'as rassurée avec sagesse, pour tes conseils, pour ta patience et pour toutes ces longues conversations au téléphone. Depuis toute petite tu es mon exemple, toujours studieuse, persévérante et dévouée. J'espère réussir ma vie, de la même façon que tu as réussi la tienne.

Merci Maéva pour ton aide, pour ta bienveillance, pour ton écoute, et pour les bons gâteaux que tu m'as préparés. J'aimerais avoir autant de courage, de générosité et de force que toi. Tu as un cœur en or, et tu es vraiment la petite sœur que tout le monde rêve d'avoir. Je te souhaite que du bonheur pour les années à venir, tu le mérites sincèrement.

Et bien sûr, merci pour toutes les corvées auxquelles j'ai échappé, grâce à vous pour réviser.

\section{À mon beau-frère, mon neveu et à ma nièce,}

Merci Willy de m'avoir encouragé toutes ces années, et toujours avec le sourire et la bonne humeur. Merci de supporter les conversations interminables que j'ai avec ma sœur, et de toujours si bien m'accueillir chez toi. Je suis très heureuse de compter dans votre famille.

Merci à mes bébés adorés, Ezio le plus beau et Néïa ma pépette ; vous être encore trop jeunes pour vous rendre compte à quel point vous êtes une source de bonheur et de motivation pour moi. Je vous aime très fort. 
THROMBOSE VEINEUSE PRÉCOCE DU GREFFON RÉNAL : QUELLE PRISE EN CHARGE? 


\section{TABLE DES MATIÈRES}

LISTE DES TABLEAUX ET FIGURES.............................................p.13

LISTE DES ABRÉVIATIONS.................................................p.14

RÉSUMÉ......................................................................... 15

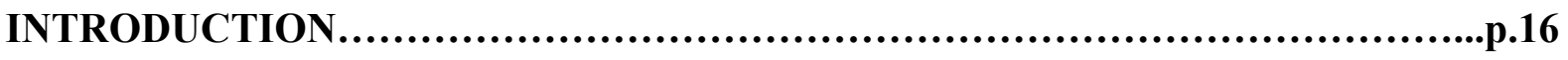

MATÉRIÉLS ET MÉTHODES.....................................................p.17

RÉSULTATS.....................................................................p.

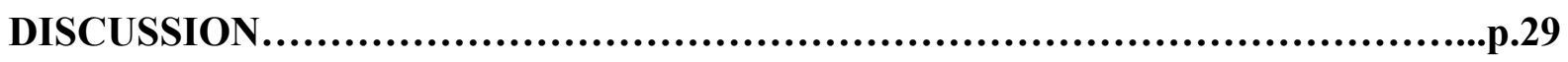

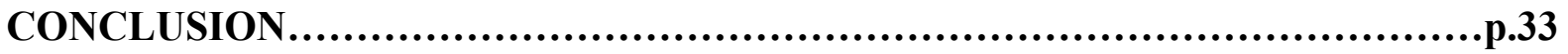

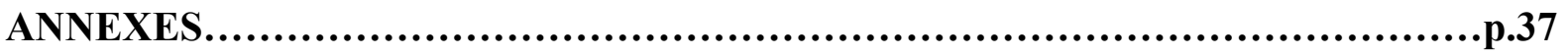

RÉFÉRENCES BIBLIOGRAPHIQUES.......................................p.42

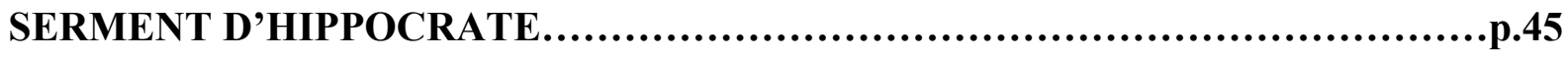

SERMENT D'HIPPOCRATE (Conseil National de l'Ordre des Médecins) ............p.46 


\section{LISTE DES TABLEAUX}

\section{TABLEAUX}

Tableau I : Caractéristiques des receveurs.

Tableau II : Caractéristiques des donneurs.

Tableau III : Circonstances de la greffe.

Tableau IV : Arguments diagnostiques de thrombose veineuse du greffon.

Tableau V : Prises en charge de la thrombose veineuse du greffon rénal.

Tableau VI : Suivi à long terme.

Tableau VII : Morbidité de la thrombose veineuse du greffon rénal.

\section{FIGURES}

Figure 1 : Diagramme de flux 


\section{LISTE DES ABRÉVIATIONS}

TV : Thrombose veineuse

CHU : Centre Hospitalo-Universitaire

IMC : Indice de Masse Corporelle

G1 : Groupe 1

G2 : Groupe 2

ATCD : Antécédents

SAL : Sérum Anti-Lymphocytaire

J0 : Jour de la transplantation

CIVD : Coagulation Intra-Vasculaire Disséminée

DVA : Donneur Vivant Apparenté

OMI : Oedèmes des Membres Inférieurs

LDH : Lactate Déshydrogénase

CPK : Créatinine Phosphokinase

ASAT : Aspartate Aminotransférase

IRM : Imagerie par Résonance Magnétique

GFS : Greffon Fonctionnel à la Sortie

IGL-1 (Institut Georges Lopez) : Solution stérile et apyrogène, destinée au rinçage et à la préservation hypothermique d'organes. 


\section{RÉSUMÉ}

\section{INTRODUCTION :}

La thrombose de la veine du greffon rénal est une complication rare (1 à $3 \%$ ) de la transplantation rénale. Elle est pourtant responsable de la perte du greffon dans la majorité des cas. Sa prise en charge tant diagnostique que thérapeutique n'est toujours pas consensuelle.

Notre étude avait pour but de comparer les différents traitements de la thrombose veineuse du greffon rénal, et leur impact sur la survie du greffon à la sortie d'hospitalisation et à 1 an.

\section{MATÉRIEL ET MÉTHODES :}

Nous avons mené une étude de cohorte rétrospective multicentrique, dans 6 centres de transplantation rénale adulte: CHU de Clermont-ferrand, CHU de Grenoble, CHU ParisNecker, CHU de Toulouse, CHU de Lyon (HEH) et CHU de Strasbourg. Les patients ont été identifiés à partir de la base prospective Cristal de l'agence de biomédecine. Ainsi sur 11172 transplantations rénales, 182 thromboses ont été observées $(1,6 \%)$. Deux patients ont été exclus des analyses par manque de données, et quatre par incertitude diagnostique.

\section{RÉSULTATS :}

Les patients de notre étude étaient majoritairement des hommes (56.2\%), âgés en moyenne de 55 ans, recevant une première transplantation rénale. Trente-quatre patients $(19,3 \%)$ avaient des antécédents de thrombose. Le greffon était issu de donneur dit marginal, dans $51.2 \%$ des cas.

Nous avons observé 16 thromboses veineuses peropératoires (G1) et 160 postopératoires (G2). Tous les patients à l'exception d'un seul, ont bénéficié d'une prise en charge chirurgicale. À la sortie d'hospitalisation, le greffon était fonctionnel dans respectivement $62,5 \% \quad(\mathrm{G} 1)$ et $8,1 \% \quad(\mathrm{G} 2)$ des cas. Chez ces derniers, une transplantectomie pour nécrose du greffon a été réalisée d'emblée dans $59.1 \%$ des cas. En cas de reprise chirurgicale, deux techniques sont privilégiées: la thrombectomie par veinotomie de décharge et la réfection de l'anastomose veineuse. La thrombectomie par veinotomie de décharge semble être la meilleure option thérapeutique. Le pronostic est meilleur pour les thromboses veineuses tardives, survenant à partir du sixième jour postopératoire.

Les complications de la thrombose veineuse du greffon et de ses suites étaient nombreuses : 5 patients sont décédés $(2.8 \%), 5(2.8 \%)$ ont présenté un choc hémorragique, 29 (16.5\%) un hématome, $38(21.6 \%)$ une infection post-opératoire et 97 patients $(55,1 \%)$ ont bénéficié d'une transfusion sanguine.

\section{CONCLUSION :}

Notre étude confirme le pronostic très sombre de la thrombose veineuse précoce du greffon rénal. Malgré une prise en charge en urgence dès le diagnostic évoqué, elle aboutit le plus souvent à une transplantectomie. 


\section{INTRODUCTION}

En France, près de 3500 personnes bénéficient d'une transplantation rénale chaque année. Alors que la survie rénale à 1 an est de près de 91,4\% (1), cette intervention n'est toutefois pas dénuée de risques notamment chirurgicaux. Les complications vasculaires de la greffe rénale, peuvent atteindre près de $7 \%$ des transplantations (2). Il peut s'agir de thrombose artérielle ou veineuse, de sténose artérielle, de dissection artérielle ou encore de la constitution d'un pseudo-anévrysme (3).

La thrombose de la veine du transplant, est une complication grave dont la fréquence est estimée entre 0,1 à $4,2 \%$ des greffes (4)(5). Elle survient dans la majorité des cas, dans les 7 premiers jours post-opératoires (6). Elle doit être évoquée devant des douleurs non calmées par les traitements antalgiques usuels, la survenue d'une oligo-anurie, un redon trop productif ou encore la majoration d'une hématurie macroscopique.

La suspicion clinique peut être confirmée par une échographie doppler rénale (7), un angioscanner abdominal ou encore, une uro-IRM (8)(9).

Il n'existe à ce jour aucune recommandation concernant la prise en charge de la thrombose veineuse du greffon post-transplantation rénale. Plusieurs traitements ont été rapportés dans la littérature dans des études rétrospectives: la reprise chirurgicale, avec veinotomie de décharge et thrombectomie (10), la réfection anastomotique, ou encore l'explantation, rinçage avec un soluté de conservation et réimplantation (11); le traitement endovasculaire (12) ou la thrombolyse (13). Les résultats de ces prises en charge sont souvent médiocres et conduisent le plus souvent à une transplantectomie.

Le but de notre étude était de comparer l'efficacité de ces différents traitements sur la survie du greffon. 


\section{MATÉRIELS ET MÉTHODES}

1) Population :

Une étude rétrospective multicentrique observationnelle a été réalisée dans 6 centres de transplantation rénale adulte : CHU de Clermont-Ferrand, CHU de Grenoble, CHU de Lyon HEH, CHU de Paris-Necker, CHU de Strasbourg et CHU de Toulouse.

Les patients ont été identifiés à partir de la base prospective de données Cristal de l'agence de biomédecine. Etaient inclus, les patients âgés de plus de 18 ans ayant bénéficié d'une transplantation rénale entre le 01/01/1997 et le 31/12/2020, compliquée d'une thrombose veineuse du greffon lors de l'hospitalisation initiale.

Étaient recueillies, à partir du dossier patient, les données démographiques, cliniques et biologiques du receveur telles que : l'âge, le sexe, l'indice de masse corporel (IMC), les antécédents thromboemboliques, les pathologies hématologiques, les antécédents de fausses couches, le tabagisme, le diabète, la maladie rénale initiale, la présence d'un traitement anticoagulant ou antiagrégant avant greffe, et les traitements immunosuppresseurs d'induction. Nous avons également recueilli à partir de la base de données Cristal, les caractéristiques démographiques et cliniques du donneur suivantes: type de donneur (donneur vivant ou décédé), son âge, donneur dit « marginal » avec des critères de sélection étendus (14), la présence de facteurs de risques thrombo-emboliques, l'année de la greffe, et les conditions de prélèvement.

Les données per-opératoires de la greffe étaient collectées à partir des comptes rendus opératoires. Nous distinguions, le type de greffe (bi-greffe, greffe multi-organe, ou transplantation rénale seule), les durées d'ischémie froide et chaude, le côté de la greffe, la latéralité du rein, et à partir des comptes rendus opératoires les éventuelles anomalies anatomiques, l'existence d'anastomoses difficiles, et le rinçage ou non des vaisseaux en peropératoire. Nous avons également relevé les signes évocateurs de la thrombose de la veine du greffon rénal, cliniques et ou biologiques, et les imageries permettant de le confirmer; ainsi que leurs prises en charge, leurs résultats fonctionnels à terme, et les complications secondaires à ces prises en charge. 
2) Définition des groupes :

Sur les 11172 patients greffés rénaux de 1997 à 2020, au sein de ces 6 centres, 182 ont présenté une thrombose de la veine du greffon rénal. Étaient exclus 2 patients avec des thromboses partielles, 2 patients pour lesquels le diagnostic n'était pas formel et 2 patients par manque de données.

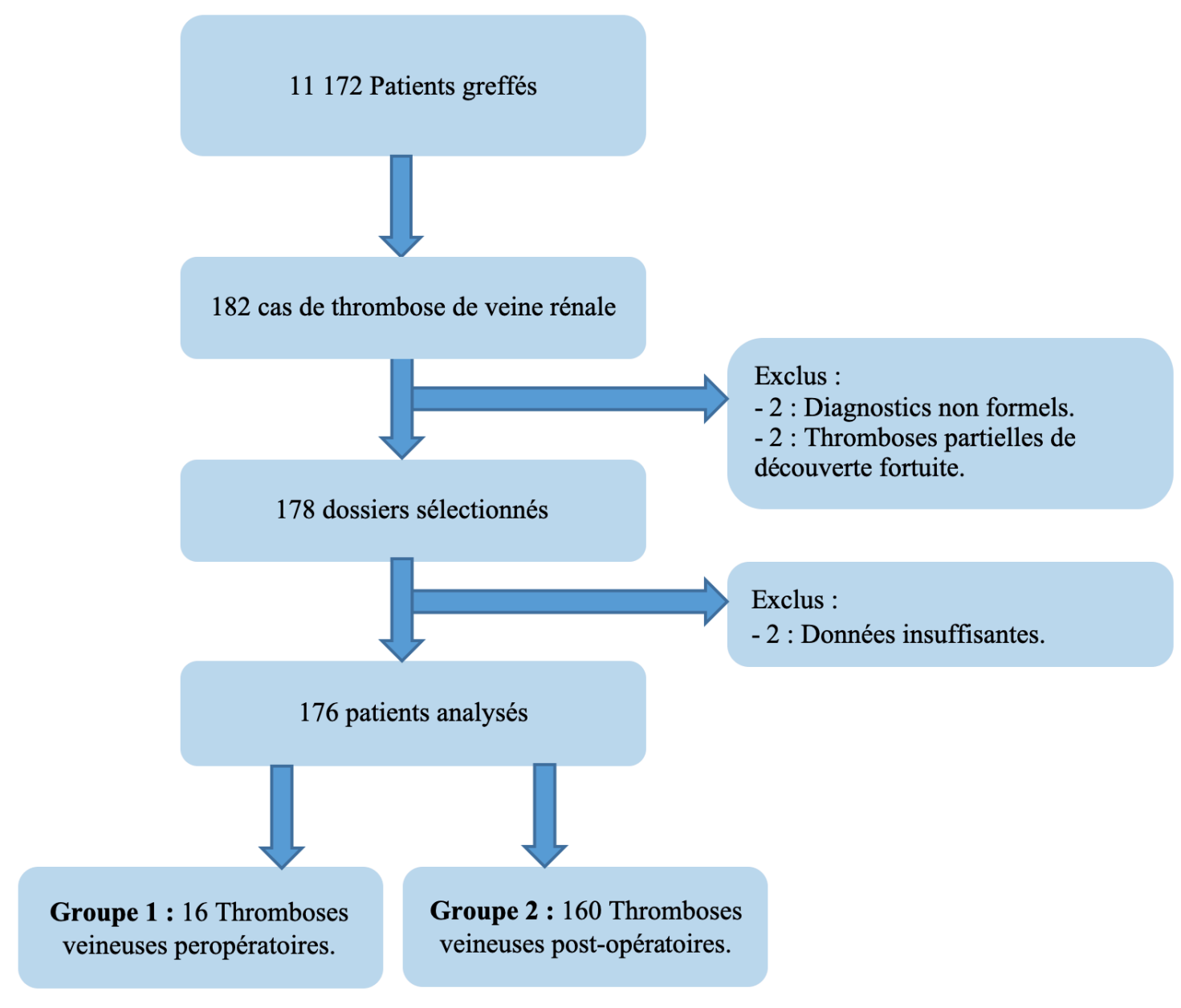

Figure 1 : Diagramme de flux. Patients greffés dans les 6 différents centres, de 1997 à 2020.

Les patients ont été divisés en 2 groupes. Le groupe $1(\mathrm{G} 1 ; \mathrm{N}=16)$ incluant les patients ayant présentés une thrombose de la veine rénale peropératoire. Le Groupe $2(\mathrm{G} 2 ; \mathrm{N}=160)$ comprend les patients dont le diagnostic de thrombose veineuse était diagnostiqué en postopératoire. 
2) Analyses statistiques :

L'analyse statistique est principalement de nature descriptive. Les données catégorielles sont décrites par des effectifs et pourcentages, alors que les données quantitatives sont présentées, au regard de leur distribution statistique, en termes de moyenne et écart-type ou médiane et intervalle interquartile. La normalité (distribution gaussienne) a été étudiée par le test de Shapiro-Wilk. Les relations entre variables de nature catégorielle ont été analysées par test exact de Fisher. Les analyses ont été réalisées avec le logiciel Stata (version 15, StataCorp, College Station, USA). Tous les tests statistiques ont été réalisés en formulation bilatérale pour un risque d'erreur de première espèce de $5 \%$. 


\section{RÉSULTATS}

a) Caractéristiques de la population :

Les patients inclus dans notre étude étaient majoritairement des hommes 99/176 (56.2\%), âgés de 56,5 ans \pm 10 ans, dont l'IMC moyen était de $26,2 \pm 3 \mathrm{~kg} / \mathrm{m}^{2}$. Sur les 176 patients, trente et un étaient diabétiques $(17.2 \%)$ et $16(9,5 \%)$ présentaient un tabagisme actif. Un antécédent de thrombose était rapporté chez 34 patients (19\%) et pour 12 d'entre eux il s'agissait de thromboses multiples $(6,8 \%)$. Une pathologie hématologique ou immunologique à risque de thrombose était retrouvée dans $9,1 \%$ des cas $(16 / 176)$.

Trente-huit patients $(21,6 \%)$ étaient sous traitement anti-aggrégant au long cours, et 13/176 $(7,3 \%)$ sous anticoagulant (Tableau I). 


\begin{tabular}{|c|c|}
\hline & Cohorte $(\mathrm{N}=176)$ \\
\hline \multicolumn{2}{|l|}{ Facteurs de risques cardiovasculaires } \\
\hline Âge $(\mathrm{N}=176)$ & $56,5[45,5-65]$ \\
\hline Hommes & $99 / 176(56,2 \%)$ \\
\hline IMC en $\mathrm{kg} / \mathrm{m} 2(\mathrm{~N}=167)$ & $25,8[22,6-29,3]$ \\
\hline Diabète $(\mathrm{N}=176)$ & $31 / 176(17,6 \%)$ \\
\hline Tabagisme actif & $16 / 167(9,5 \%)$ \\
\hline Plaquettes à $\mathrm{J} 0$ en $\mathrm{G} / \mathrm{l}(\mathrm{N}=127)$ & $196[168-240]$ \\
\hline \multicolumn{2}{|l|}{ Facteurs de risques thrombo-emboliques } \\
\hline ATCD de thrombose veineuse & $34 / 176(19,3 \%)$ \\
\hline Thrombose de la veine sur greffon antérieur & $3 / 176(1,7 \%)$ \\
\hline Thrombose de fistule artérioveineuse & $10 / 176(5,7 \%)$ \\
\hline Thrombose veineuse profonde & $6 / 176(3,4 \%)$ \\
\hline Embolie pulmonaire & $2 / 176(1,1 \%)$ \\
\hline Thrombose de cathéter central & $1 / 176(0,6 \%)$ \\
\hline Multiples ou récidivantes & $12 / 176(6,8 \%)$ \\
\hline Pathologies favorisant les thromboses & $16 / 176(9,1 \%)$ \\
\hline Pathologies systémiques & $2 / 176(1,1 \%)$ \\
\hline Troubles de la coagulation & $9 / 176(5,1 \%)$ \\
\hline Pathologies hématologiques & $5 / 176(2,8 \%)$ \\
\hline ATCD de fausses couches & $9 / 77(11,6 \%)$ \\
\hline ATCD de varices & $6 / 173(3,5 \%)$ \\
\hline Chirurgie dans les 3 mois & $6 / 165(3,6 \%)$ \\
\hline \multicolumn{2}{|l|}{ Maladie rénale initiale } \\
\hline Glomérulopathies & $53 / 176(30,1 \%)$ \\
\hline Néphroangiosclérose & $25 / 176(14,2 \%)$ \\
\hline Polykystose rénale & $23 / 176(13,1 \%)$ \\
\hline Diabète & $21 / 176(11,9 \%)$ \\
\hline Néphropathie indéterminée & $18 / 176(10,2 \%)$ \\
\hline Uropathies malformatives & $14 / 176(7,9 \%)$ \\
\hline Néphropathie Intersticielle chronique & $13 / 176(7,4 \%)$ \\
\hline Vascularites & $4 / 176(2,3 \%)$ \\
\hline Maladies systémiques & $4 / 176(2,3 \%)$ \\
\hline Oxaliurie & $1 / 176(0,6 \%)$ \\
\hline \multicolumn{2}{|l|}{ Traitement habituel } \\
\hline Anticoagulants & $13 / 176(7,3 \%)$ \\
\hline Anti-aggrégants & $38 / 176(21,6 \%)$ \\
\hline Pillule oestroprogestative & $3 / 176(3,9 \%)$ \\
\hline \multicolumn{2}{|l|}{ Traitement immunosuppresseur } \\
\hline Traitement immunosuppresseur d'induction & $146 / 176(82,9 \%)$ \\
\hline SAL & $91 / 146(62,3 \%)$ \\
\hline Basiliximab & $51 / 146(34,9 \%)$ \\
\hline Non précisé & $4 / 146(2,7 \%)$ \\
\hline Traitement initial comprenant un mTOR & $6 / 155(3,9 \%)$ \\
\hline \multicolumn{2}{|c|}{$\begin{array}{l}\boldsymbol{I M C}=\text { Indice de masse corporelle, } \boldsymbol{A T C D}=\text { Antécécdents, } \boldsymbol{S A L}=\text { Sérum antilymphocytaires } \\
\boldsymbol{J} \boldsymbol{O}=\text { Jour de la transplantation }\end{array}$} \\
\hline
\end{tabular}

\section{Tableau I : Caractéristiques des receveurs.}


Dans $84,2 \%$ des cas (144/176), il s'agissait d'une première transplantation rénale (144/176, soit 84,2\%) issue de donneurs décédés de mort encéphalique ou d'arrêt cardiocirculatoire non contrôlé (166/176 ; soit 94,3\%). L'âge médian du donneur était de 60 ans (46$71) ; 51,2 \%$ des greffons (85/166) étaient considérés comme marginaux ; et 23,9\% des donneurs (42/176) ont présenté un arrêt cardio respiratoire récupéré avant le prélèvement (Tableau II).

\begin{tabular}{|c|c|}
\hline & Cohorte $(\mathrm{N}=176)$ \\
\hline \multicolumn{2}{|l|}{ Caractéristiques des donneurs } \\
\hline Âge $(N=171)$ & $60[46-71]$ \\
\hline Critères étendus donneur & $85 / 166 \quad(51,2 \%)$ \\
\hline$>60$ ans & $81 / 166(48,8 \%)$ \\
\hline$>55$ ans avec au moins 2 facteurs de risques* & $4 / 166(2,4 \%)$ \\
\hline Arrêt cardio-respiratoire & $42 / 176(23,9 \%)$ \\
\hline CIVD & $2 / 176(1,1 \%)$ \\
\hline Ischémie froide en minutes $(\mathrm{N}=161)$ & 918 [714-1259] \\
\hline Cannulation difficile & $4 / 155(2,6 \%)$ \\
\hline DVA & $10 / 176(5,6 \%)$ \\
\hline \multicolumn{2}{|l|}{ 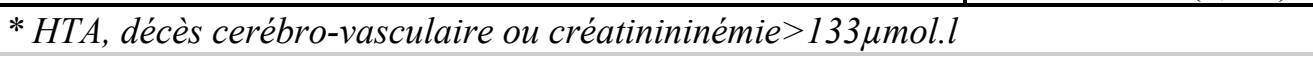 } \\
\hline \multicolumn{2}{|c|}{$\boldsymbol{C I V D}=$ Coagulation intra-vasculaire disséminée, $\boldsymbol{D V} \boldsymbol{A}=$ Donneur vivant apparenté } \\
\hline
\end{tabular}

\section{Tableau II : Caractéristiques des donneurs.}

b) Données de la transplantation rénale.

L'ischémie froide médiane était de $15 \mathrm{~h} 18 \mathrm{~min}$. L'ischémie chaude médiane était de 45 minutes. Chez 23 receveurs $(13,4 \%)$, l'anatomie veineuse du greffon était anormale (11 veines multiples, 10 plaies veineuse sur prélèvement et 2 veines dysplasiques). De plus, sept opérateurs ont repris l'anastomose veineuse lors de la greffe (4,4\%). Les vaisseaux de 37 greffons ont été rincés en peropératoire avec du sérum hépariné ou du sérum physiologique $(27,4 \%)$, et 13 patients $(9,4 \%)$ ont bénéficié d'une héparinothérapie peropératoire (Tableau III). 


\begin{tabular}{|c|c|}
\hline Caractéristiques pré-opératoires & \\
\hline Première greffe rénale & $144 / 171(84,2 \%)$ \\
\hline Type de greffe & \\
\hline Rein unique & $163 / 176(93 \%)$ \\
\hline Bigreffe & $8 / 176(4 \%)$ \\
\hline Greffe multi-organes & $5 / 176(3 \%)$ \\
\hline Greffe Homolatérale & $75 / 146(51,4 \%)$ \\
\hline Greffe Controlatérale & $71 / 146(48,6 \%)$ \\
\hline Rein droit & $101 / 175(57,7 \%)$ \\
\hline Caractéristiques per opératoires & \\
\hline Ischémie chaude en minutes $(\mathrm{N}=103)$ & $45[34-55]$ \\
\hline Anomalie sur la veine & $23 / 172(13,4 \%)$ \\
\hline Veines multiples dont une au moins sacrifiée & $11 / 172(6,4 \%)$ \\
\hline Plaies sur prélèvement & $10 / 172(5,8 \%)$ \\
\hline Veines dysplasiques & $2 / 172(1,2 \%)$ \\
\hline Reprise de l'anastomose veineuse & $7 / 159(4,4 \%)$ \\
\hline Twist de la veine rénale & $1 / 159(0,6 \%)$ \\
\hline Thrombus partiel & $2 / 159(1,3 \%)$ \\
\hline Etranglement de la veine illiaque & $1 / 159(0,6 \%)$ \\
\hline Déchirure de l'anastomose sur poussée abdominale & $1 / 159(0,6 \%)$ \\
\hline Non précisé & $2 / 159(1,3 \%)$ \\
\hline Anomalie anatomie artérielle & $55 / 170(32,3 \%)$ \\
\hline Artères multiples & $37 / 170(21,8 \%)$ \\
\hline Plaque d'athérome & $14 / 170(8,2 \%)$ \\
\hline Plaies sur prélèvement & $2 / 170(1,2 \%)$ \\
\hline Non précisé & $2 / 170(1,2 \%)$ \\
\hline Héparinothérapie per-opératoire & $13 / 138(9,4 \%)$ \\
\hline Rinçage des vaisseaux & $37 / 135(27,4 \%)$ \\
\hline
\end{tabular}

\section{Tableau III : Circonstances de la greffe.}

c) Diagnostic de la thrombose veineuse

Dans la majorité des cas, la thrombose veineuse était symptomatique (86.2\%). Elle se manifestait par une oligo-anurie $(63,1 \%)$, une douleur anormale sur la loge rénale $(26,9 \%)$, une hématurie franche $(17,5 \%)$. La thrombose veineuse s'est compliqué de troubles hémodynamiques, définis par la nécessité de remplissage avec du sérum physiologique ou la mise en place d'amines vasopressives chez 14 patients $(8,6 \%)$ et d'un choc hémorragique chez 7 autres receveurs (4,3\%). Dans le groupe 2, le principal critère biologique ayant conduit 
au diagnostic était la majoration de la créatininémie $(81 / 160 ; 50,6 \%)$. Pour les patients du groupe 2, 68/160(42,5\%) patients ont bénéficié d'une échographie doppler du greffon, 22 $(13,8 \%)$ d'un angioscanner abdominopelvien, $61(38,1 \%)$ patients des deux, et $6(3,7 \%)$ ont bénéficié d'une échographie associée à une IRM. (Tableau IV).

\begin{tabular}{|c|c|}
\hline \multirow{3}{*}{$\begin{array}{l}\text { Délai } \\
\text { Thrombose yeineuse Per-onératoire }\end{array}$} & \\
\hline & \\
\hline & $16 / 176(9,1 \%)$ \\
\hline Thrombose veineuse post-opératoire & $160 / 176(90,9 \%)$ \\
\hline Délai moyen entre greffe et diagnostic en heures $(\mathrm{N}=\mathbf{1 6 0})$ & $48[24-120]$ \\
\hline \multicolumn{2}{|l|}{ Critères cliniques } \\
\hline Choc hémorragique & $7 / 160(4,3 \%)$ \\
\hline Troubles hémodynamiques & $14 / 160(8,6 \%)$ \\
\hline Douleur anormale & $43 / 160(26,9 \%)$ \\
\hline Majoration de l'hématurie macroscopique & $28 / 160(17,5 \%)$ \\
\hline Oligo-anurie & $101 / 160(63,1 \%)$ \\
\hline Fièvre & $3 / 160(1,9 \%)$ \\
\hline Redon productif & $6 / 160(3,7)$ \\
\hline Autres (OMI, douleur testiculaire) & $3 / 160(1,9 \%)$ \\
\hline Asymptomatique & $22 / 160(13,75 \%)$ \\
\hline \multicolumn{2}{|l|}{ Critères biologiques ayant conduit au diagnostic } \\
\hline Aucun & $37 / 160(23,1 \%)$ \\
\hline Hyperlactatémie & $10 / 160(6,3 \%)$ \\
\hline Augmentation des LDH & $17 / 160(10,6 \%)$ \\
\hline Augmentation de la créatinininémie & $81 / 160(50,6 \%)$ \\
\hline Anémie & $5 / 160(3,1 \%)$ \\
\hline Thrombopénie & $6 / 160(3,7 \%)$ \\
\hline Augmentation des CPK & $2 / 160(1,2 \%)$ \\
\hline Augmentation des ASAT & $1 / 160(<1 \%)$ \\
\hline Autres* & $5 / 160(3,1 \%)$ \\
\hline \multicolumn{2}{|l|}{ Critères radiologiques } \\
\hline Aucun & $3 / 160(1,9 \%)$ \\
\hline Echographie-doppler seule & $68 / 160(42,5 \%)$ \\
\hline Angioscanner abdominopelvien seul & $22 / 160(13,8 \%)$ \\
\hline Echographie Doppler et IRM & $6 / 160(3,7 \%)$ \\
\hline Echographie doppler + angioscanner abdominopelvien & $61 / 160(38,1 \%)$ \\
\hline Thromboses concomittantes & $44 / 176(25 \%)$ \\
\hline Embolie pulmonaire & $10 / 176(5,7 \%)$ \\
\hline Thrombose veineuse profonde & $13 / 176(7,4 \%)$ \\
\hline Thrombose de l'artère du greffon & $13 / 176(7,4 \%)$ \\
\hline Thrombose de fistule artério-veineuse & $8 / 176(4,5 \%)$ \\
\hline \multicolumn{2}{|c|}{ *3 syndromes inflammatoires, 2 hyperkaliémies } \\
\hline \multicolumn{2}{|c|}{$\begin{array}{l}\text { OMI }=\text { Oedèmes des membres inférieurs } ; \boldsymbol{L D H}=\text { Lactate Déshydrogénase } ; \boldsymbol{C P K}=\text { Créatinine } \\
\text { Phosphokinase } ; \boldsymbol{A S A T}=\text { Aspartate Aminotransférase } ; \boldsymbol{I R M}=\text { Imagerie par Résonance } \\
\text { Magnétique. }\end{array}$} \\
\hline
\end{tabular}

Tableau IV : Arguments diagnostiques de thrombose veineuse du greffon. 
d) Prises en charge thérapeutiques.

Dans le groupe $1(\mathrm{G} 1=16), 10 / 16$ thromboses veineuses peropératoires $(62,5 \%)$ ont été traitées chirurgicalement par une réfection de l'anastomose veineuse, 6/16 (37,5\%) par une thrombectomie avec veinotomie de décharge. Quatre reprises chirurgicales (4/16 25\%) ont abouties à une transplantectomie peropératoire, et $1 / 16(6,3 \%)$ à une transplantectomie secondaire. Douze reprises chirurgicales (12/16, 75\%) semblaient satisfaisantes selon le chirurgien, mais 1 d'entre elle a abouti à une transplantectomie secondaire (Tableau V).

Dans le groupe $2(\mathrm{G} 2=160)$, le délai de survenue de la thrombose veineuse était de $48 \mathrm{~h}$ [24-120]. La prise en charge était quasi-exclusivement chirurgicale 159/160 (99,4\%), à l'exception d'un patient qui a bénéficié d'une héparinothérapie seule. Chez 94/159 patients $(59,1 \%)$, une transplantectomie a été réalisée d'emblée devant un greffon rénal nécrosé. Pour les 65 autres patients $(65 / 159 ; 40,9 \%)$, les 2 techniques chirurgicales principalement utilisées étaient la thrombectomie par veinotomie de décharge et la réfection de l'anastomose veineuse, plus ou moins associée à un rinçage du greffon. Pour un patient, le type de reprise chirurgicale n'était pas précisé.

La reprise chirurgicale s'est accompagnée d'un échec de procédure primaire pour 23/159 $(14,5 \%)$ des cas ; une tentative de revascularisation était réalisée, mais ne permettait pas une recoloration suffisante, entraînant une transplantectomie dans le même temps opératoire. Pour 42 patients $(42 / 159 ; 26,4 \%)$, la reprise chirurgicale était satisfaisante selon le chirurgien, avec une revascularisation convenable du greffon. Mais parmi eux, une transplantectomie a été réalisée secondairement, pendant l'hospitalisation, chez 22 patients $(22 / 159 ; 13,8 \%)$. (Tableau V).

Les différentes prises en charge sont illustrés en annexe. 


\begin{tabular}{|c|c|c|c|c|}
\hline & \multicolumn{4}{|c|}{ GREFFON FONCTIONNEL À LA SORTIE } \\
\hline & \multicolumn{2}{|c|}{ TV peropératoires $(\mathrm{N}=16)$} & \multicolumn{2}{|c|}{ TV post-opératoires $(\mathrm{N}=160)$} \\
\hline & $\mathbf{N}(\%)$ & p & $\mathbf{N}(\%)$ & $\mathbf{p}$ \\
\hline & $10 / 16(62,5 \%) i$ & $\mathrm{p}<0.001$ & $13 / 160(8,1 \%)$ & $p<0.001$ \\
\hline Délai moyen de survenue de la TV & $\mathbf{N}=16$ & - & $\mathbf{N}=159$ & - \\
\hline$=0 \mathrm{H}$ & $10 / 16(62,5 \%) !$ & - & - & - \\
\hline$<24 \mathrm{H}$ & $\begin{array}{ll}- & 1\end{array}$ & - & $1 / 23(4,3 \%)$ & $p=0.004$ \\
\hline $24-48 \mathrm{H}$ & - & - & $1 / 55(1,8 \%)$ & $P=0.004$ \\
\hline $48 \mathrm{H}-6$ jours & - & - & $3 / 47(6,4 \%)$ & $p=0.004$ \\
\hline$>6$ jours & - & - & $8 / 34(23,5 \%)$ & $p=0.004$ \\
\hline Héparinothérapie & $\mathbf{N}=16$ & - & $\mathbf{N}=160$ & - \\
\hline Seule & 0 & - & $0 / 1$ & - \\
\hline Héparinothérapie Per-opératoire sur la reprise & $5 / 8(62,5 \%)$ & - & $5 / 24(20,8 \%)$ & - \\
\hline Héparinothérapie Post-opératoire à la reprise & $0 / 2$ & - & $1 / 10(10 \%)$ & - \\
\hline Reprise chirurgicale & $\mathbf{N}=\mathbf{1 6}$ & - & $\mathbf{N}=159$ & - \\
\hline Reprise chirurgicale satisfaisante & $10 / 12(90,9 \%)$ & - & $13 / 42(30,9 \%)$ & - \\
\hline Transplantectomie secondaire & $0 / 1$ & - & $0 / 22$ & - \\
\hline Echec du sauvetage, et transplantectomie concomittante & $0 / 4$ & - & $0 / 23$ & - \\
\hline Transplantectomie d'emblée & 0 & - & $0 / 94$ & - \\
\hline Type de reprise chirurgicale & $\mathbf{N}=\mathbf{1 6}$ & - & $N=64$ & - \\
\hline Thrombectomie par veinotomie de décharge & $3 / 6(50 \%)$ & $p=0.61$ & $10 / 37(27 \%)$ & $p=0.34$ \\
\hline Rinçage du greffon à l'IGL-1 & 0 & - & $0 / 1$ & - \\
\hline Rinçage du greffon au sérum hépariné & 0 & - & $0 / 1$ & - \\
\hline Réfection anastomose veineuse & $7 / 10(70 \%)$ & $p=0.61$ & $3 / 22(13,6 \%)$ & $p=0.34$ \\
\hline Rinçage du greffon à l'IGL-1 & 0 & - & $1 / 1(100 \%)$ & - \\
\hline Explantation, rinçage, ré-implantation & 0 & - & $0 / 2$ & - \\
\hline Evacuation d'hématome de la loge du greffon & 0 & - & $0 / 2$ & - \\
\hline Pose d'une prothèse annelée & 0 & - & $0 / 1$ & - \\
\hline Sonde de Fogarty sur extention de la thrombose en fémoral & $0 / 2$ & - & $0 / 5$ & - \\
\hline
\end{tabular}

\section{Tableau V : Prises en charge.}

e) Survie rénale après thrombose de la veine du greffon.

La survie rénale en sortie d'hospitalisation était de 13,1\% (23/176). Elle était différente si la thrombose survenait en per opératoire (G1) ou post opératoire (G2) respectivement de $10 / 16(62,5 \%)$, dans le groupe G1 et de $13 / 160(8,1 \%)$ dans le groupe $\mathrm{G} 2$, avec $p<0.001$. 
Dans le G2, sur les 42 reprises chirurgicales satisfaisantes, il y a eu 22 transplantectomies secondaires $(22 / 160 ; 12,5 \%)$. Parmi les 20 greffons restants, seulement 13 greffons étaient fonctionnels à la sortie. Huit de ces 13 greffons, correspondaient à des thromboses survenues après le $6^{\text {ème }}$ jour de la greffe. Parmi les 7 non fonctionnels, 2 patients sont décédés en postopératoire, 3 greffons ont étés transplantectomisé plusieurs mois après la greffe, 1 greffon a été embolisé et le dernier a été laissé en place. Concernant le type de prise en charge, 10/37 (27\%) thrombectomies par veinotomie de décharge ont permis de sauver le greffon, contre $3 / 22(13,6 \%)$ réfections d'anastomose veineuse $(\mathrm{p}=0.34)$. Les autres prises en charge n'ont pas été efficaces.

\section{Greffons sauvés $(\mathrm{N}=\mathbf{2 3})$}

Créatinine à 1 an en $\mu$ mol.1 $(\mathrm{N}=22)$

Créatinine à 5 ans en $\mu$ mol.l $(\mathrm{N}=16)$

Greffons perdus $(\mathbf{N}=\mathbf{1 5 3})$

Nouvelle greffe pour les greffons perdus $(\mathrm{N}=151)$

Délai nouvelle greffe en mois $(\mathrm{N}=43)$

\begin{tabular}{|c|}
\hline Cohorte $(\mathrm{N}=176)$ \\
\hline $155[130-207]$ \\
\hline $179[126-230]$ \\
\hline $44(29,1 \%)$ \\
\hline $24[17-36]$ \\
\hline
\end{tabular}

\section{$\underline{\text { Tableau VI : Suivi à long terme. }}$}

Pour les 23 greffons fonctionnels à la sortie d'hospitalisation, la créatininémie était satisfaisante à un an : 155 $\mu$ mol.1 [130-207].

Seize patients sur les 23 (69.6\%) gardaient un greffon fonctionnel à 5 ans, 2 patients étaient dialysés, 1 patient est décédé et 4 étaient perdus de vue.

De plus 44/153 (29,1\%) des patients ayant perdu leur greffon ont pu bénéficier d'une nouvelle greffe. (Tableau VII)

f) Complications de la thrombose veineuse du greffon et de sa prise en charge.

Cinq patients $(5 / 176 ; 2,8 \%)$ sont décédés au décours de la prise en charge, dont 4/176 $(2,3 \%)$ dans les 15 premiers jours de la transplantation. Dans le G1, 1 patient est décédé à J4 
d'un choc hémorragique; et 4 sont décédés dans le G2 : 1 décès au bloc opératoire sur un choc hémorragique au cours d'une transplantectomie, 1 décès à J6 sur anémie et hyperkaliémie, les 2 autres, à $\mathrm{J} 11$ et à $\mathrm{J} 61$ étaient liés à une défaillance multiviscérale, précédée de multiples reprises chirurgicales.

Au total 5 patients $(5 / 176 ; 2,8 \%)$ ont présenté un choc hémorragique, dont 3 ont étés contrôlés. La majorité des patients de notre cohorte ont présenté une perte sanguine significative définie par la nécessité d'au moins une transfusion de culots globulaires $(97 / 176 ; 55,1 \%) ; 38$ ont présenté une infection post-opératoires $(38 / 176 ; 21,6 \%)$, et 29 ont développé un volumineux hématome (29/176; 16,5\%). (Tableau VII).

La durée médiane de l'hospitalisation était de 14[9-22] jours.

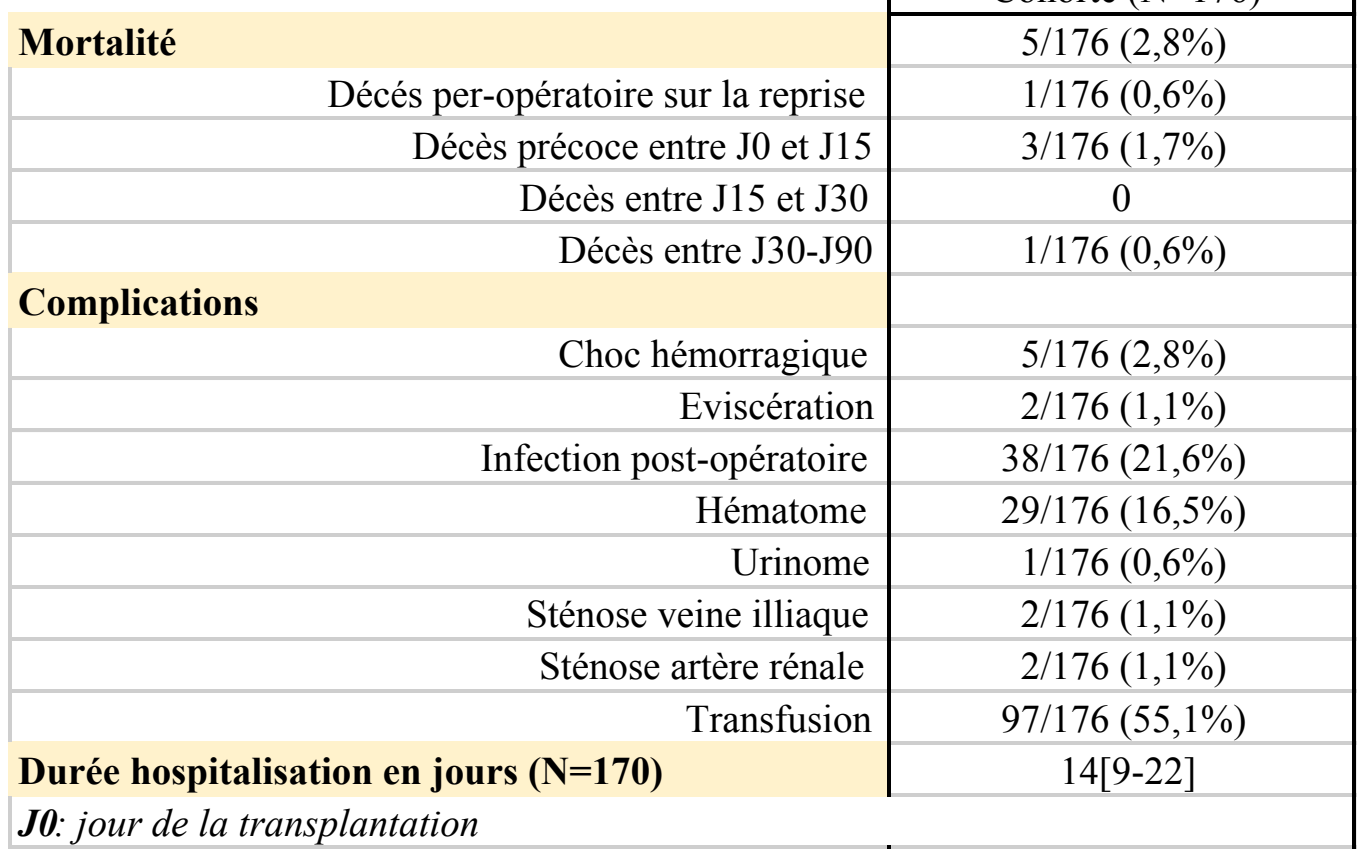

Tableau VII : Morbidité de la thrombose veineuse du greffon rénal. 


\section{DISCUSSION}

Cette étude est à notre connaissance, la plus grande série de thrombose veineuse de greffon rénal. Nous avons rapporté une incidence de thrombose veineuse de 1,6\%. Ce taux est comparable avec les données de la littérature. (15)

Dans notre cohorte, la thrombose veineuse est responsable d'une perte du greffon dans $91,9 \%$ des cas. Seule une thrombose per opératoire est associée à une meilleure survie du greffon $(63,5 \%)$, ce qui est probablement dû à la possibilité d'une prise en charge immédiate (11).

Que la thrombose soit peropératoire ou post-opératoire, la prise en charge a été presque exclusivement chirurgicale. En effet dans notre cohorte, un seul patient a été traité par une anticoagulation sans succès. Dans la littérature, un seul cas de traitement par héparine à dose curative avec préservation de la fonction du greffon (16) a été rapporté, chez un patient ayant présenté une thrombose veineuse du greffon tardive, plus de 9 ans après la transplantation. L'utilisation de l'héparine dans notre cohorte, semble peu fréquente, tant en préventif lors de la transplantation $(9,4 \%)$ ou de la reprise chirugicale (15\%), qu'en curatif en post-opératoire de celle-ci $(6,3 \%)$. Probablement que ce taux est lié au risque hémorragique majoré en postopératoire immédiat (17).

Deux études de cas américaines (18)(19), détaillaient la prise en charge efficace par thrombolyse de deux thromboses veineuse du greffon, mais cette technique n'a pas été utilisée dans notre cohorte. Il en est de même pour le traitement endovasculaire. Pourtant une étude française de 1999 (12) exposait deux cas de thrombose de veine du greffon rénal, traitées efficacement par thombo-aspiration associée à une héparinothérapie à doses curatives, avec un greffon fonctionnel dans les deux cas. La thrombo-aspiration associée à la thrombolyse in situ, était également satisfaisante dans l'étude de cas de Melamed M.L (20). Mais ces procédures endovasculaires restent risquées sur des sutures chirurgicales récentes, ce qui explique leur faible utilisation en pratique courant sur les thromboses veineuses du greffon.

Les deux techniques de revascularisation les plus utilisées étaient la thrombectomie par veinotomie de décharge et la réfection anastomotique. Face à une thrombose veineuse 
peropératoire, la réfection anastomotique est la technique la plus favorisée. Ceci est le plus souvent justifié par une imperfection chirurgicale à l'origine de cette thrombose, nécessitant une réfection complète de l'anastomose : un twist sur la veine, un étranglement de la veine iliaque, une plicature de la veine en positionnant le greffon, un repli sur une longue veine, une disparité de calibre entre les vaisseaux du greffon et ceux du receveur, ou encore une compression externe (3).

Huit des 13 greffons fonctionnels à la sortie concernaient des thromboses veineuses survenues après J6. Une des hypothèses pour expliquer ce résultat, serait de considérer que la formation de ses thromboses est plus insidieuse, et permet une meilleure reprise de fonction du greffon. La littérature évoque aussi les états thrombogènes comme étiologie de ces thromboses veineuses (21). Dans notre cohorte, 19,3\% des patients présentaient un antécédent de thrombose, 9,1\% des pathologies potentiellement thrombogènes, et 3,6\% une chirurgie récente. De plus, on a diagnostiqué une thrombose concomitante chez $25 \%$ des patients. On a observé que parmi les patients ayant un greffon sauvé, il y avait moins de thromboses concomitantes $(2 / 23 ; 8,5 \%)$ et d'antécédent de thrombose veineuse $(3 / 23 ; 13 \%)$ que pour les patients ayant perdus leur greffon (respectivement $42 / 153 ; 27,4 \%$ et $31 / 153 ; 20,2 \%$ ). Cela pourrait se justifier par une survenue plus brutale des thromboses veineuses liées à un état thrombogène, avec un pronostic plus sombre.

Les 2 explantations complètes du greffon avec rinçage et ré-implantation n'ont pas étés efficaces dans cette étude, contrairement aux résultats d'une série de 5 cas de complications veineuses pour lesquelles les greffons ont étés explantés reperfusés et retransplanté, avec un résultat satisfaisant pour tous les patients. Toutefois, un seul patient avait présenté une thrombose de veine, et l'âge moyen des donneurs était bien plus bas, à 39 ans (22). Dans notre étude, les 3 greffons ayant étés rincé avec de l'IGL-1 ou du sérum hépariné sur la reprise chirurgicale, étaient fonctionnels en sortie d'hospitalisation

La morbidité reste majeure suite à la survenue d'une thrombose veineuse. En effet dans notre recueil nous avons observé $2,8 \%$ de décès, dont $80 \%$ survenus dans les 15 premiers jours de la greffe. Les autres complications observées étaient sévères : $2,8 \%$ de chocs hémorragiques, $21,6 \%$ infections post-opératoire et $55,1 \%$ des patients ont nécessité une transfusion sanguine. Celle-ci peut limiter l'accès à une nouvelle greffe du fait de l'immunisation contre le système antigénique leucocytaire humain (23). Toutefois 29,1\% 
patients ayant perdu leur greffon, ont pu bénéficier d'une nouvelle greffe rénale, avec un délai médian d'un an.

Notre travail présente plusieurs limites. Tout d'abord, nous rapportons les résultats d'une cohorte rétrospective. Ainsi certaines difficultés au cours du prélèvement du rein ou de la transplantation ont pu être méconnues. Par ailleurs lors de la reprise chirurgicale, 94 transplantectomies ont été réalisées devant un greffon nécrotique. Il est possible qu'il y ait eu un retard au diagnostic et/ou à leur prise en charge. En effet les signes cliniques de thromboses veineuses sont aspécifiques (douleurs, oligo-anurie, hématurie macroscopique) mais doivent faire évoquer le diagnostic (4). La surveillance de la créatinine et des LDH sériques systématiquement dans les premiers jours pourraient être une aide à la surveillance (24).

De plus, le délai de prise en charge n'a pas été relevé dans notre étude. Mais devant un probable doute à l'échographie (25), 41,8 \% des patients ont bénéficié d'une autre imagerie (angioscanner ou IRM) ce qui prolonge le délai de prise en charge. On a donc évalué la survie du greffon en fonction du nombre d'examen radiologique réalisé. Sur les 67 patients dans le groupe 2 qui ont eu deux examens radiologiques, 5 greffons ont été sauvés soit 7,5\% ; et pour les 93 qui n'en ont eu qu'un seul ou aucun, 8 greffons ont été sauvés soit $8,6 \%$, ce qui reste comparable. Dans notre étude, le délai de reprise chirurgicale prolongé par la réalisation de deux examens radiologiques n'a pas eu d'impact sur la survie du greffon. Bien que peu connus, les facteurs pronostiques de la thrombose veineuse semblent être multiples, et complexes.

Une autre limite de notre étude est l'absence de groupe contrôle nous empêchant d'étudier les facteurs de risques associés à la survenue de la thrombose veineuse du greffon. Dans une revue de la littérature, Keller et al (26) ont rapportés les facteurs de risques suivants : les donneurs décédés âgés de plus de 60 ans ou de moins de 6 ans, la survenue d'un trouble hémodynamique péri-opératoire chez le receveur, un antécédent de thrombose, et/ou de diabète chez le receveur. Les greffons dit marginaux, représentaient environ $51 \%$ de notre cohorte, ce qui est comparable avec les données de l'agence de biomédecine française, qui rapporte 45,8\% de greffons marginaux sur l'ensemble des greffes. Une étude de 2009, comparant les complications de la transplantation rénale, entre les greffons standard et les greffons marginaux, ne retrouvait pas de différence (27). 
Enfin, la littérature évoque également un risque majoré de thrombose en cas rein droit (28) (29), la veine à droite étant plus courte, et la plastie de la veine cave, pouvant engendrer la création d'un espace mort, et favoriser cette complication. Dans notre cohorte, nous avons $58 \%$ de reins droit. El Zorkany (4) évoquait en 2015, un risque majoré de thrombose veineuse pour les greffes controlatérales (reins droits à gauche et inversement), par compression de l'artère. Cela n'a pas été retrouvé dans notre étude, puisque nous avions 48,6\% de greffe controlatérales.

On observe à ce jour, une grande variabilité dans le traitement de la thrombose veineuse du greffon rénale. Ce travail pourrait-être à l'origine de l'élaboration de protocoles de prises en charges et de reprises chirurgicales codifiées, reproductibles d'un centre à l'autre. 


\section{CONCLUSION}

Notre étude confirme l'extrême sévérité de la thrombose veineuse précoce du greffon rénal, responsable d'une perte de greffon dans la très grande majorité des cas, notamment en cas de thrombose post-opératoire. Bien que d'un pronostic réservé, sa prise en charge est très majoritairement chirurgicale en France et repose sur une thrombectomie par veinotomie de décharge.

Des études complémentaires doivent nous permettre de mieux identifier les patients à risque de thrombose veineuse, afin d'assurer une surveillance rapprochée, et d'envisager d'élaborer des protocoles de thromboprophylaxie adaptée.

Pierre Clavelou

Doyen-Directeur

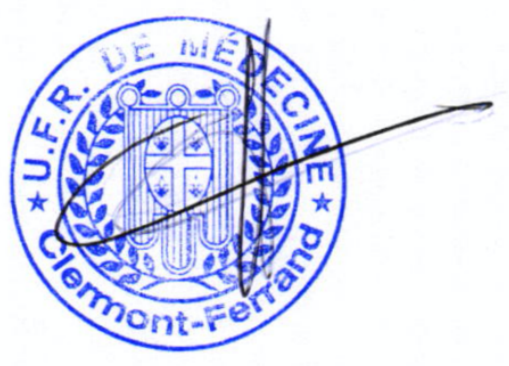

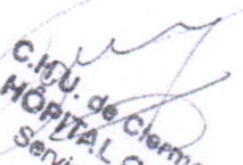

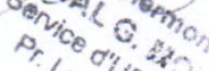

of 


\section{ANNEXES}

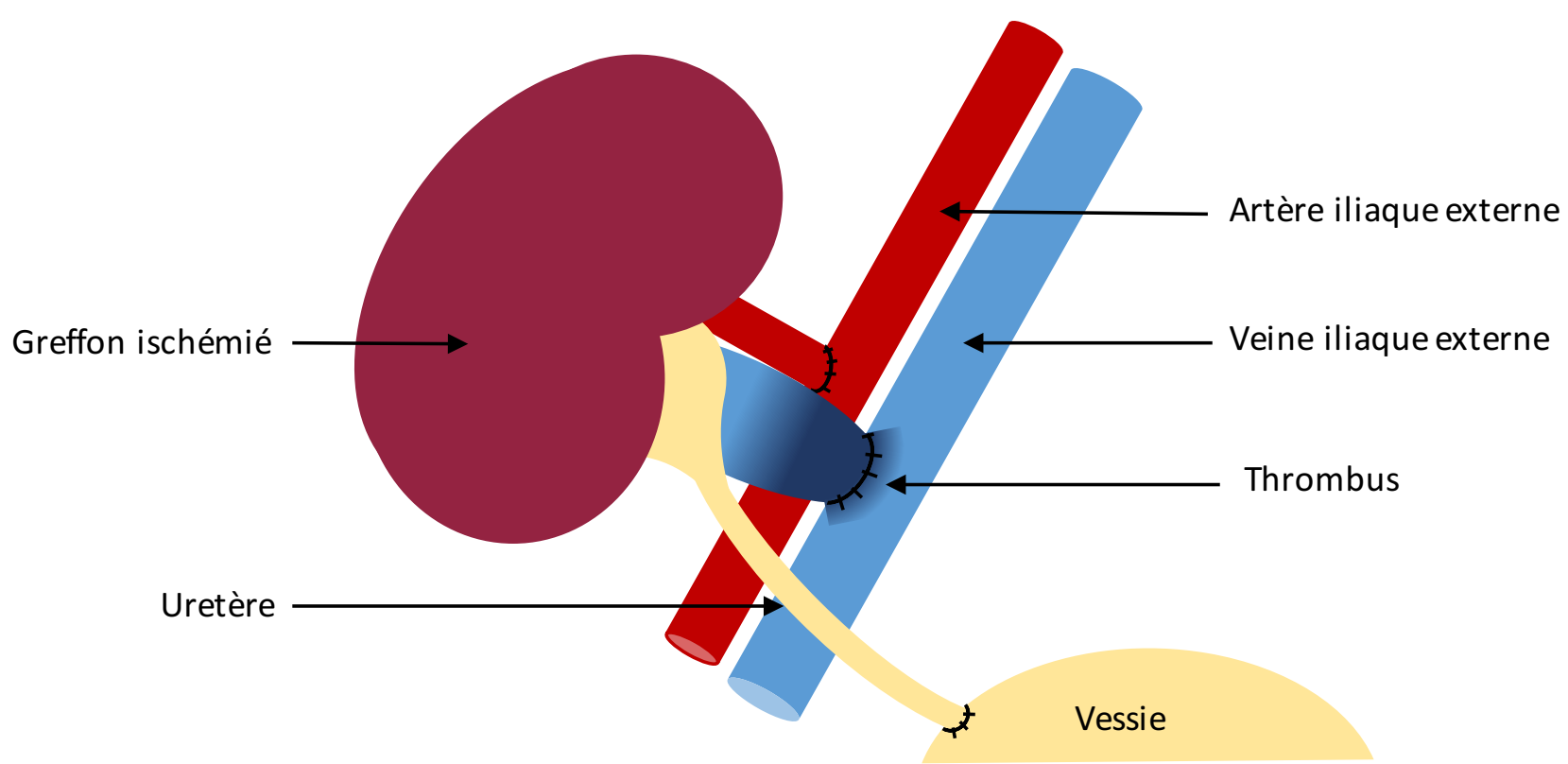

Annexe 1 : Thrombose veineuse du greffon rénal. 


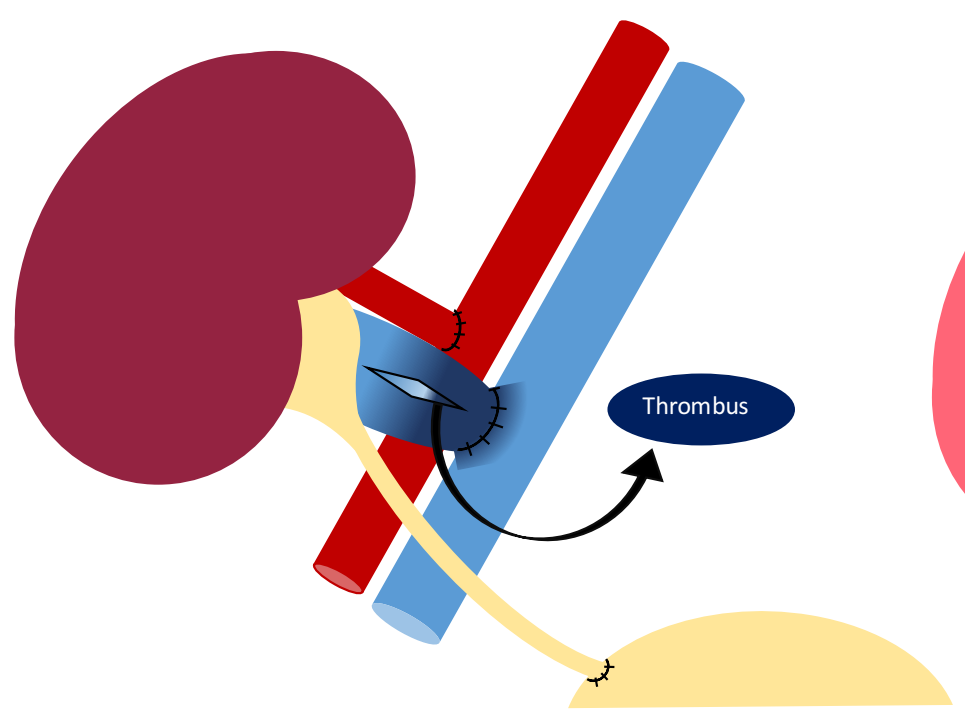

1.

Annexe 2: Thrombectomie par veinotomie de décharge.
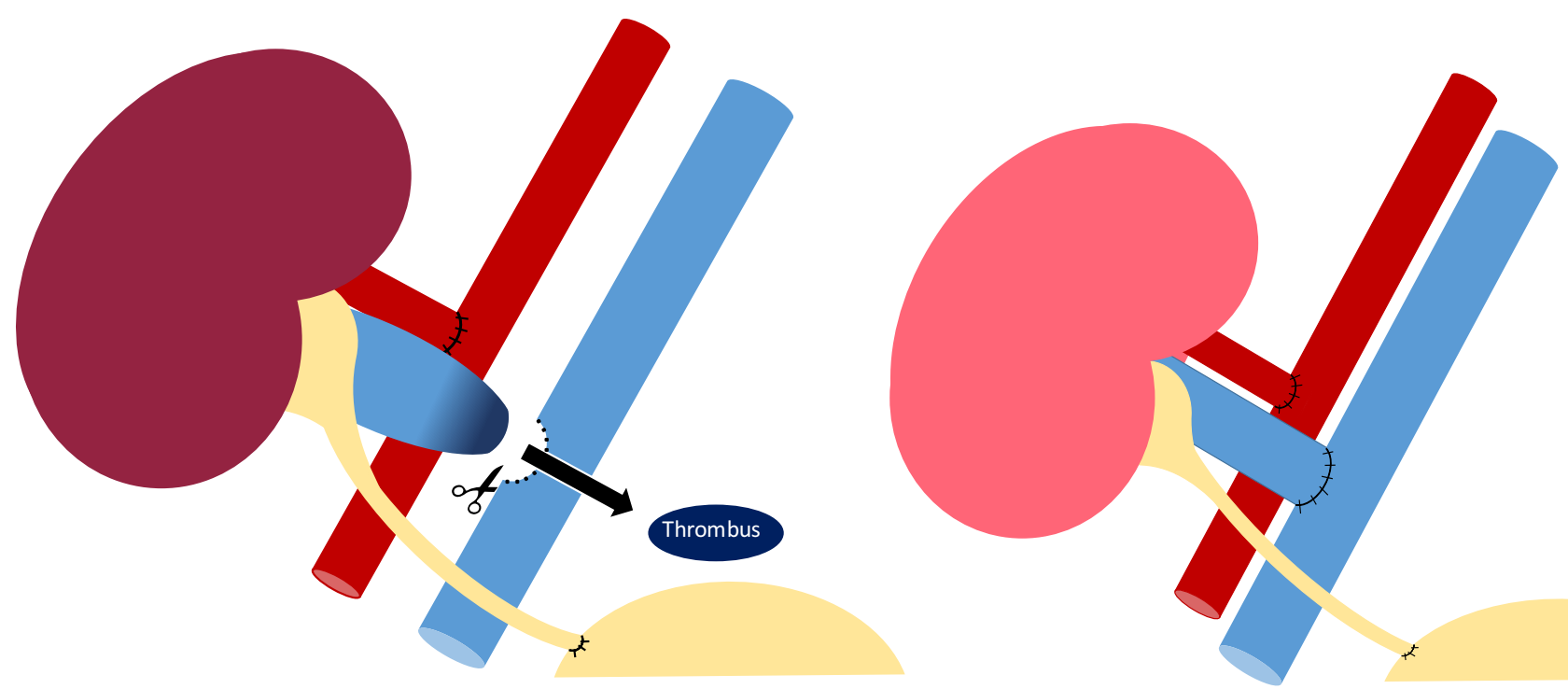

1.

$\underline{\text { Annexe } 3 \text { : Réfection de l'anastomose veineuse. }}$

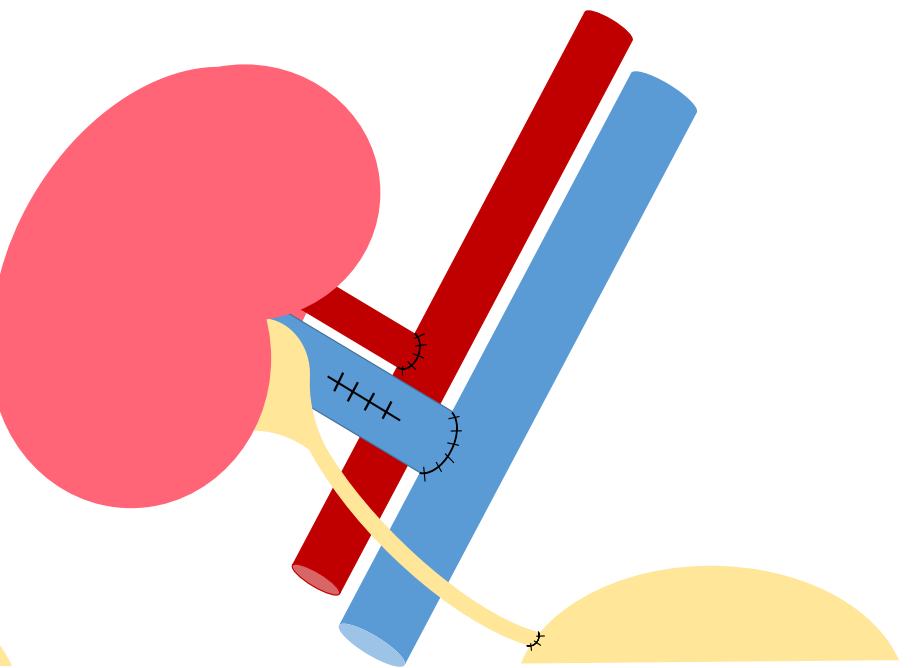

2. 


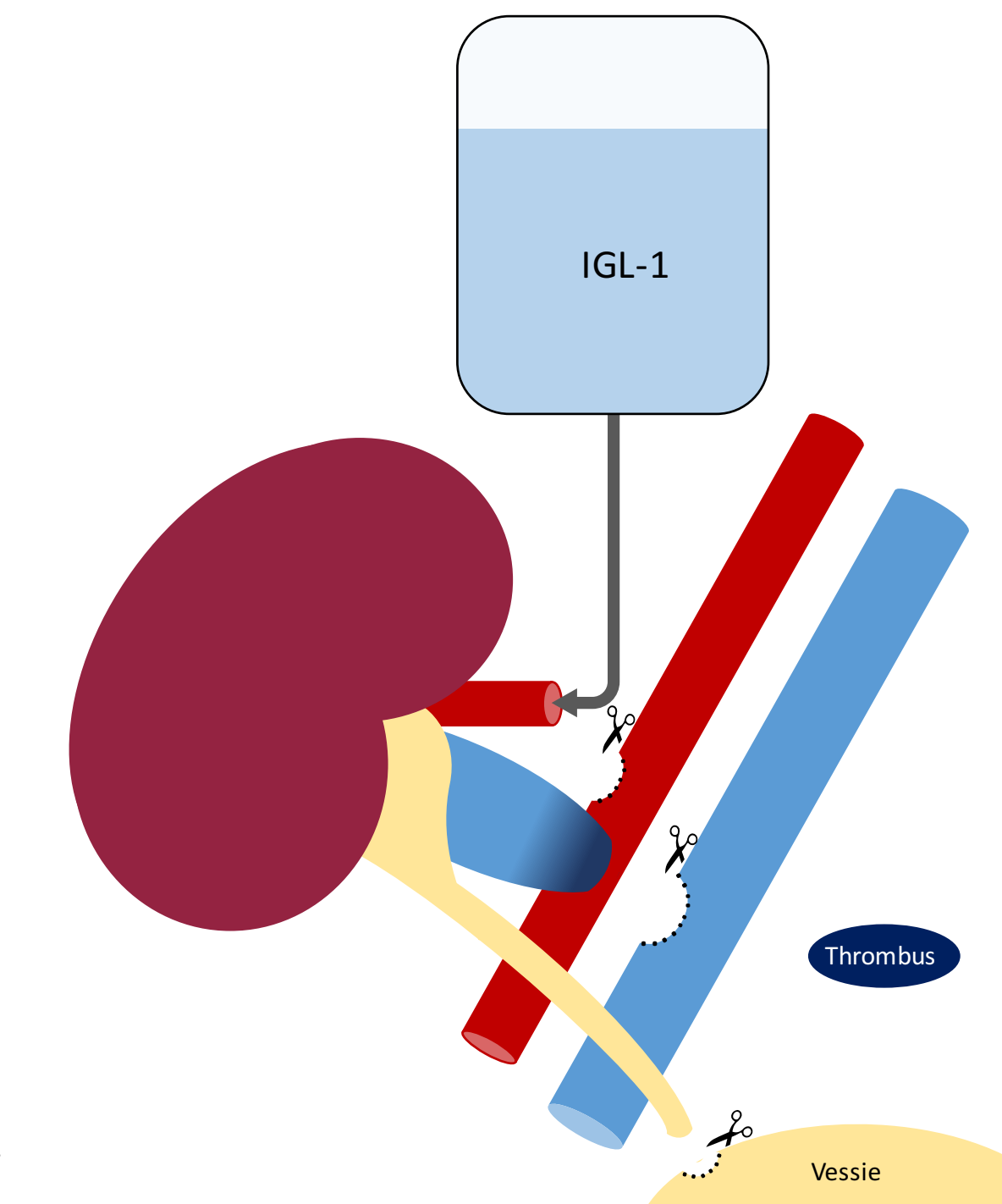

1.

2.

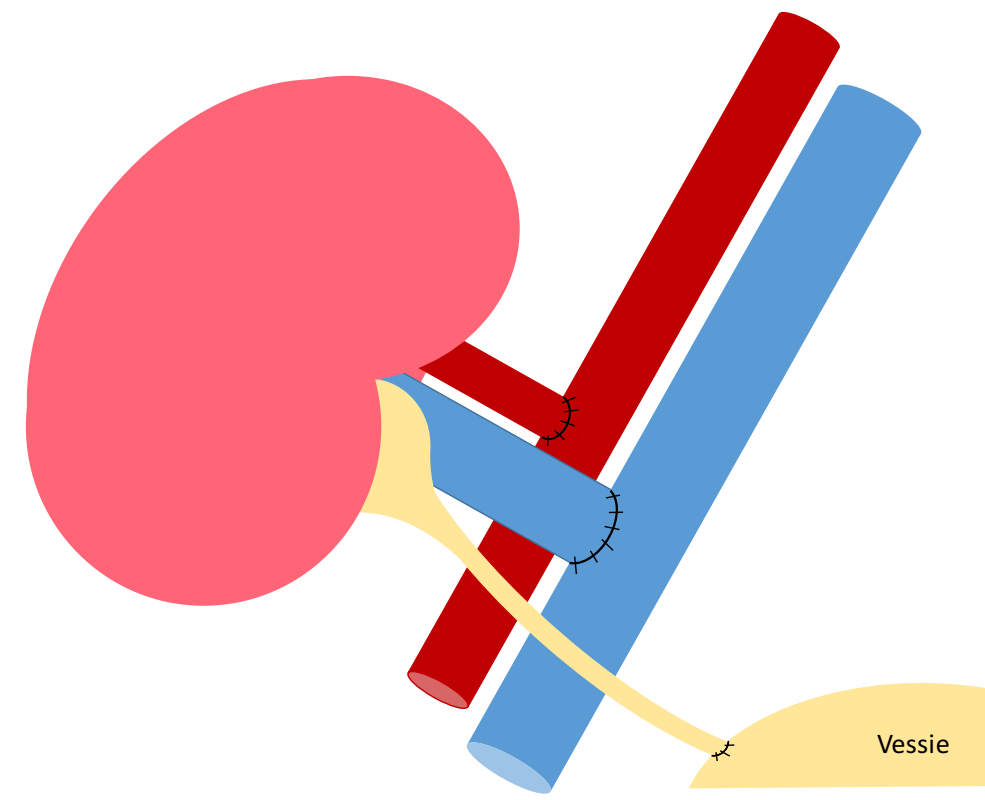

$\underline{\text { Annexe } 4 \text { : Explantation, rinçage à l'IGL et réimplantation. }}$ 


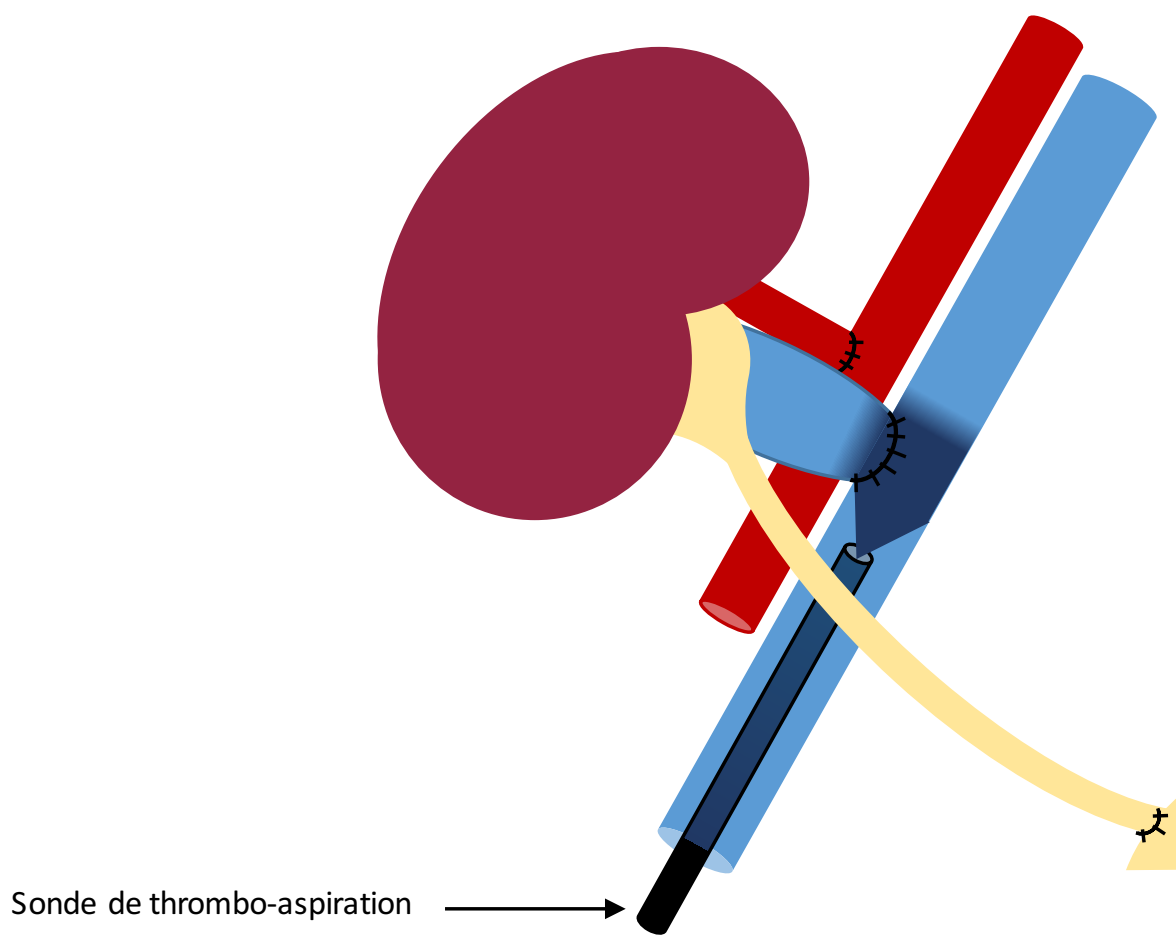

Annexe 5: Thromboaspiration par cathétérisme de la veine fémorale.

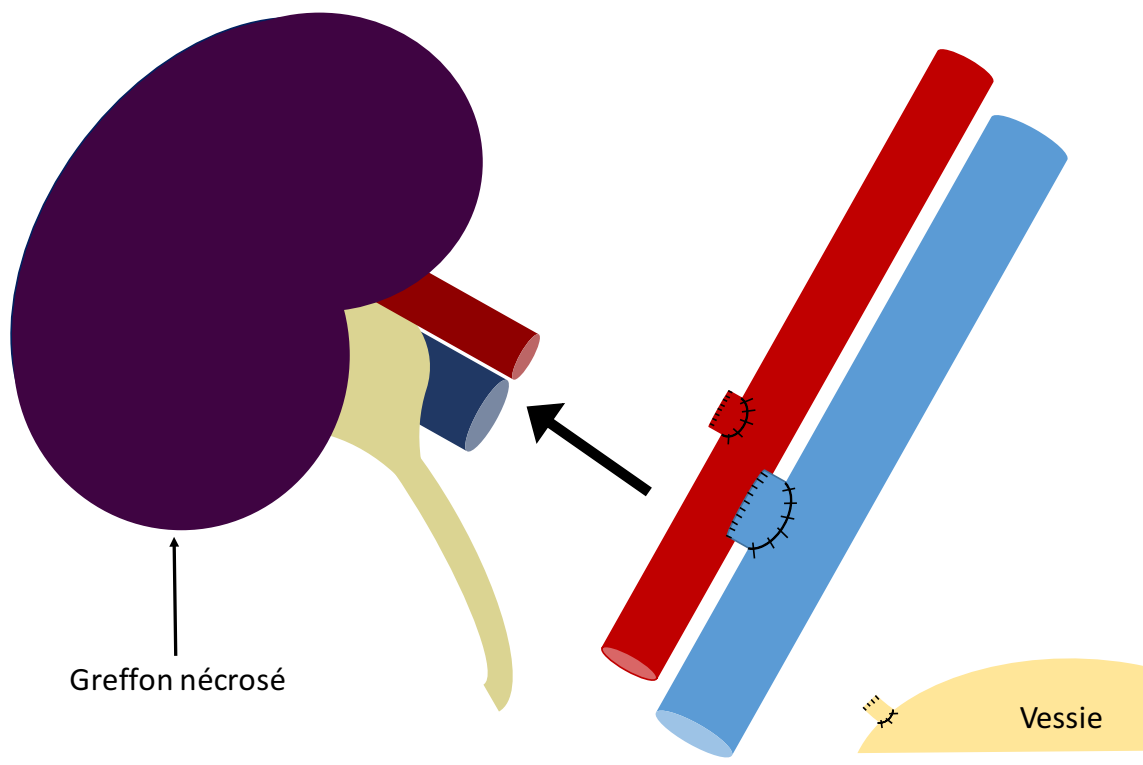

$\underline{\text { Annexe } 6 \text { : Transplantectomie. }}$ 


\begin{tabular}{|c|}
\hline \multicolumn{1}{|c|}{ Définition des donneurs aux critères élargis } \\
\hline Âge $>60$ ans. \\
Âge de 50 à 59 ans avec $\geq 2$ facteurs de risques suivant : \\
- Antécédent d'hypertension artérielle \\
- Décès cérébro-vasculaire \\
- Creatinine $>153 \mu$ mol..l. \\
\hline
\end{tabular}

Annexe 7 : Critères étendus donneur. 


\section{RÉFÉRENCES BIBLIOGRAPHIQUES}

1 - «https://www.agence-biomedecine.fr/annexes/bilan2016/donnees/organes/06-rein/synthese.htm », s. d.

2 - Orlic, P, D Vukas, I Drescik, A Ivancic, G Blecic, B Budiselic, G Velcic, et al. « Vascular Complications after 725 Kidney Transplantations during 3 Decades ». Transplantation Proceedings $35, \mathrm{n}^{\mathrm{O}} 4$ (juin 2003): 1381-84.

3 - Julia, P., J.-M. Alsac, et J.-N. Fabiani. « Complications vasculaires de la transplantation rénale ». EMC - Techniques chirurgicales - Chirurgie vasculaire 6, $\mathrm{n}^{\circ} 4$ (janvier 2011): 1-16.

4 - El Zorkany, Khaled, Julie-Michelle Bridson, Ajay Sharma, et Ahmed Halawa. « Transplant Renal Vein Thrombosis ». Experimental and Clinical Transplantation: Official Journal of the Middle East Society for Organ Transplantation 15, n 22 (avril 2017): 123-29.

5 - Eufrásio, P., B. Parada, P. Moreira, P. Nunes, S. Bollini, A. Figueiredo, et A. Mota. « Surgical Complications in 2000 Renal Transplants ». Transplantation Proceedings 43, $\mathrm{n}^{\circ} 1$ (février 2011): $142-44$.

6 - Aschwanden, Markus, Christoph Thalhammer, Stefan Schaub, Thomas Wolff, Jürg Steiger, et Kurt A. Jaeger. « Renal vein thrombosis after renal transplantation - early diagnosis by duplex sonography prevented fatal outcome ». Nephrology Dialysis Transplantation 21, $\mathrm{n}^{\circ} 3$ (1 mars 2006): 825-26.

7 - Sadej, Piotr, Rick I. Feld, et Adam Frank. « Transplant Renal Vein Thrombosis: Role of Preoperative and Intraoperative Doppler Sonography ». American Journal of Kidney Diseases 54, n 6 (décembre 2009): 1167-70.

8 - Ardelean, A., D. Mandry, et M. Claudon. « Complications vasculaires de la transplantation rénale : conduite diagnostique ». Journal de Radiologie 92, nº 4 (avril 2011): 343-57.

9- Sugi, Mark D., Gayatri Joshi, Kiran K. Maddu, Nirvikar Dahiya, et Christine O. Menias. « Imaging of Renal Transplant Complications throughout the Life of the Allograft: Comprehensive Multimodality Review ». RadioGraphics 39, n 5 (septembre 2019): 1327-55.

10 - Renoult, Edith, Luc Cormier, Michel Claudon, Than Cao-Huu, Luc Frimat, Olivier Gaucher, Jacques Hubert, et Michèle Kessler. « Successful Surgical Thrombectomy of Renal Allograft Vein Thrombosis in the Early Postoperative Period ». American Journal of Kidney Diseases 35, $\mathrm{n}^{0} 5$ (1 mai 2000): e21.1-e21.4.

11 - Kawano, Paulo Roberto, Hamilto Akihissa Yamamoto, Rodrigo Gerra, Paula Dalsoglio Garcia, Mariana Moraes Contti, Hong Si Nga, Henrique Mochida Takase, Ariane Moyses Bravin, et Luis Gustavo Modelli de Andrade. «A case report of venous thrombosis after kidney transplantation - We can save the graft? Time is the success factor ». International Journal of Surgery Case Reports 36 (19 mai 2017): 82-85.

12 - Rerolle, Jean Philippe, Corinne Antoine, Alain Raynaud, Bernard Beyssen, Pierre Julia, Alain Duboust, et Denis Glotz. " Successful Endoluminal Thrombo-Aspiration of Renal Graft Venous Thrombosis ». Transplant International 13, $\mathrm{n}^{\circ} 1$ (janvier 2000): 82-86. 
13 - Schwieger, J., R. Reiss, J. L. Cohen, L. Adler, et D. Makoff. « Acute Renal Allograft Dysfunction in the Setting of Deep Venous Thrombosis: A Case of Successful Urokinase Thrombolysis and a Review of the Literature ». American Journal of Kidney Diseases: The Official Journal of the National Kidney Foundation 22, n ${ }^{\circ} 2$ (août 1993): 345-50.

14 - Metzger, Robert A., Francis L. Delmonico, Sandy Feng, Friedrich K. Port, James J. Wynn, et Robert M. Merion. «Expanded Criteria Donors for Kidney Transplantation: Expanded Criteria Donors ». American Journal of Transplantation 3 (avril 2003): 114-25.

15 - Tavakkoli, Mahmoud, Reza Zafarghandi, Rahim Taghavi, Alireza Ghoreifi, et Masoud Zafarghandi. «Immediate Vascular Complications After Kidney Transplant: Experience from 2100 Recipients ». Experimental and clinical transplantation: official journal of the Middle East Society for Organ Transplantation, 2 décembre 2016.

16 - Ferreira, C., L. Pereira, P. Pereira, I. Tavares, S. Sampaio, M. Bustorff, et M. Pestana. « Late Allograft Renal Vein Thrombosis Treated With Anticoagulation Alone: A Case Report ». Transplantation Proceedings 48, ${ }^{\circ} 9$ (novembre 2016): 3095-98.

17 - A. Schwein, A. Lejay, M. Ohana, M. Sagnard, F. Thaveau, N. Chak, P. Wolf, Y. Georg, Complications vasculaires de la transplantation rénale, EMC Techniques chirugicales - Chirurgie vasculaire, 2020.

18 - Robinson, Jeff M., Charles H. Cockrell, Jaime Tisnado, Michael C. Beachley, Marc P. Posner, et Thomas F. Tracy. « Selective Low-Dose Streptokinase Infusion in the Treatment of Acute Transplant Renal Vein Thrombosis ». CardioVascular and Interventional Radiology 9, $\mathrm{n}^{\circ} 2$ (1 mars 1986): 86-89.

19 - Schwieger, J., R. Reiss, J. L. Cohen, L. Adler, et D. Makoff. « Acute Renal Allograft Dysfunction in the Setting of Deep Venous Thrombosis: A Case of Successful Urokinase Thrombolysis and a Review of the Literature ». American Journal of Kidney Diseases: The Official Journal of the National Kidney Foundation 22, nº 2 (août 1993): 345-50.

20 - Melamed, Michal L., Hyun S. Kim, Bernard G. Jaar, Ernesto Molmenti, Mohamed G. Atta, et Milagros D. Samaniego. « Combined Percutaneous Mechanical and Chemical Thrombectomy for Renal Vein Thrombosis in Kidney Transplant Recipients ». American Journal of Transplantation 5, $\mathrm{n}^{\circ}$ 3 (2005): 621-26.

21 - Englesbe, Michael J., Jeffery D. Punch, Douglas R. Armstrong, Juan D. Arenas, Randall S. Sung, et John C. Magee. « Single-Center Study of Technical Graft Loss in 714 Consecutive Renal Transplants ». Transplantation 78, $\mathrm{n}^{\circ} 4$ (27 août 2004): 623-26.

22 - Janousek, L, M Kudla, Janka Slatinska, Ondrej Viklický, et Jiri Fronek. « Kidney transplant early venous complications managed by reperfusion and re-transplantation - salvage procedure ». Bratislavské lekárske listy 115 (7 mars 2014): 101-2.

23 - Freist, Marine, Dominique Bertrand, Elodie Bailly, Céline Lambert, Paul Olivier Rouzaire, Richard Lemal, Julien Aniort, Matthias Büchler, Anne Elisabeth Heng, et Cyril Garrouste. « Management of Immunosuppression After Kidney Transplant Failure: Effect on Patient Sensitization ». Transplantation Proceedings, décembre 2020. 
24 - Bourgault, Marie, Philippe Grimbert, Catherine Verret, Jacques Pourrat, Michel Herody, Jean Michel Halimi, Alexandre Karras, et al. « Acute Renal Infarction: A Case Series ». Clinical Journal of the American Society of Nephrology 8, $\mathrm{n}^{\mathrm{o}} 3$ (7 mars 2013): 392-98.

25 - Como, Giuseppe, Jacopo Da Re, Gian Luigi Adani, Chiara Zuiani, et Rossano Girometti. « Role for contrast-enhanced ultrasound in assessing complications after kidney transplant ». World Journal of Radiology 12, $\mathrm{n}^{\circ} 8$ (28 août 2020): 156-71.

26 - Keller, Anna Krarup, Troels Munch Jorgensen, et Bente Jespersen. «Identification of Risk Factors for Vascular Thrombosis May Reduce Early Renal Graft Loss: A Review of Recent Literature ». Review Article. Journal of Transplantation. Hindawi, 31 mai 2012.

27 - Domagala, P., A. Kwiatkowski, M. Wszola, J. Czerwinski, K. Cybula, J. Trzebicki, et A. Chmura. « Complications of Transplantation of Kidneys From Expanded-Criteria Donors ». Transplantation Proceedings 41, $\mathrm{n}^{\mathrm{o}} 8$ (octobre 2009): 2970-71.

28 - Bakir, N., W. J. Sluiter, R. J. Ploeg, W. J. van Son, et A. M. Tegzess. « Primary Renal Graft Thrombosis ». Nephrology, Dialysis, Transplantation: Official Publication of the European Dialysis and Transplant Association - European Renal Association 11, n 1 (janvier 1996): 140-47.

29 - Amézquita, Y., C. Méndez, A. Fernández, S. Caldes, J. Pascual, A. Muriel, F.J. Burgos, R. Marcén, et J. Ortuño. « Risk Factors for Early Renal Graft Thrombosis: A Case-Controlled Study in Grafts From the Same Donor ». Transplantation Proceedings 40, nº 9 (novembre 2008): 2891-93. 


\section{SERMENT D'HIPPOCRATE}

En présence des Maîtres de cette FACULTE et de mes chers CONDISCIPLES, je promets et je jure d'être fidèle aux lois de l'Honneur et de la Probité dans l'exercice de la Médecine.

Je donnerai mes soins gratuits à l'indigent et je n'exigerai jamais un salaire au-dessus de mon travail. Admis dans l'intérieur des maisons, mes yeux ne verront pas ce qui s'y passe, ma langue taira les secrets qui me seront confiés et mon état ne servira pas à corrompre les mœurs ni à favoriser le crime.

Respectueux et reconnaissant envers mes MAÎTRES, je rendrai à leurs enfants l'instruction que j'ai reçue de leurs pères.

Que les HOMMES m'accordent leur estime si je suis fidèle à mes promesses. Que je sois couvert d'OPPROBRE et méprisé de mes confrères si j'y manque. 


\section{SERMENT D'HIPPOCRATE}

\section{(Conseil National de l'Ordre des Médecins)}

Au moment d'être admis à exercer la médecine, je promets et je jure d'être fidèle aux lois de l'honneur et de la probité.

Mon premier souci sera de rétablir, de préserver ou de promouvoir la santé dans tous ses éléments, physiques et mentaux, individuels et sociaux.

Je respecterai toutes les personnes, leur autonomie et leur volonté, sans aucune discrimination selon leur état ou leurs convictions. J'interviendrai pour les protéger si elles sont affaiblies, vulnérables ou menacées dans leur intégrité ou leur dignité. Même sous la contrainte, je ne ferai pas usage de mes connaissances contre les lois de l'humanité.

J'informerai les patients des décisions envisagées, de leurs raisons et de leurs conséquences. Je ne tromperai jamais leur confiance et n'exploiterai pas le pouvoir hérité des circonstances pour forcer les consciences.

Je donnerai mes soins à l'indigent et à quiconque me les demandera. Je ne me laisserai pas influencer par la soif du gain ou la recherche de la gloire.

Admis(e) dans l'intimité des personnes, je tairai les secrets qui me seront confiés. Reçu(e) à l'intérieur des maisons, je respecterai les secrets des foyers et ma conduite ne servira pas à corrompre les mœurs.

Je ferai tout pour soulager les souffrances. Je ne prolongerai pas abusivement les agonies. Je ne provoquerai jamais la mort délibérément.

Je préserverai l'indépendance nécessaire à l'accomplissement de ma mission. Je n'entreprendrai rien qui dépasse mes compétences. Je les entretiendrai et les perfectionnerai pour assurer au mieux les services qui me seront demandés.

J'apporterai mon aide à mes confrères ainsi qu'à leurs familles dans l'adversité.

Que les hommes et mes confrères m'accordent leur estime si je suis fidèle à mes promesses ; que je sois déshonoré(e) et méprisé(e) si j’y manque. 
CAMBOU Ludivine

THROMBOSE VEINEUSE PRÉCOCE DU GREFFON RÉNAL : QUELLE PRISE EN CHARGE ?

Thèse de médecine

- Faculté de médecine de Clermont-Ferrand -

Année 2021

\section{RÉSUMÉ :}

\section{INTRODUCTION :}

La thrombose de la veine du greffon rénal est une complication rare (1 à 3\%) de la transplantation rénale. Elle est pourtant responsable de la perte du greffon dans la majorité des cas. Sa prise en charge tant diagnostique que thérapeutique n'est toujours pas consensuelle.

Notre étude avait pour but de comparer les différents traitements de la thrombose veineuse du greffon rénal, et leur impact sur la survie du greffon à la sortie d'hospitalisation et à 1 an.

\section{MATÉRIEL ET MÉTHODES :}

Nous avons mené une étude de cohorte rétrospective multicentrique, dans 6 centres de transplantation rénale adulte: CHU de Clermont-ferrand, CHU de Grenoble, CHU Paris-Necker, CHU de Toulouse, CHU de Lyon (HEH) et CHU de Strasbourg. Les patients ont été identifiés à partir de la base prospective Cristal de l'agence de biomédecine. Ainsi sur 11172 transplantations rénales, 182 thromboses ont été observées (1,6\%). Deux patients ont été exclus des analyses par manque de données, et quatre par incertitude diagnostique.

\section{RÉSULTATS :}

Les patients de notre étude étaient majoritairement des hommes (56.2\%), âgés en moyenne de 55 ans, recevant une première transplantation rénale. Trente-quatre patients $(19,3 \%)$ avaient des antécédents de thrombose. Le greffon était issu de donneur dit marginal, dans $51.2 \%$ des cas.

Nous avons observé 16 thromboses veineuses peropératoires (G1) et 160 post-opératoires $(\mathrm{G} 2)$. Tous les patients à l'exception d'un seul, ont bénéficié d'une prise en charge chirurgicale. À la sortie d'hospitalisation, le greffon était fonctionnel dans respectivement $62,5 \%(\mathrm{G} 1)$ et $8,1 \%(\mathrm{G} 2)$ des cas. Chez ces derniers, une transplantectomie pour nécrose du greffon a été réalisée d'emblée dans $59.1 \%$ des cas. En cas de reprise chirurgicale, deux techniques sont privilégiées : la thrombectomie par veinotomie de décharge et la réfection de l'anastomose veineuse. La thrombectomie par veinotomie de décharge semble être la meilleure option thérapeutique. Le pronostic est meilleur pour les thromboses veineuses tardives, survenant à partir du sixième jour post-opératoire.

Les complications de la thrombose veineuse du greffon et de ses suites étaient nombreuses: 5 patients sont décédés $(2.8 \%), 5(2.8 \%)$ ont présenté un choc hémorragique, 29 (16.5\%) un hématome, $38(21.6 \%)$ une infection post-opératoire et 97 patients $(55,1 \%)$ ont bénéficié d'une transfusion sanguine.

\section{CONCLUSION :}

Notre étude confirme le pronostic très sombre de la thrombose veineuse précoce du greffon rénal. Malgré une prise en charge en urgence dès le diagnostic évoqué, elle aboutit le plus souvent à une transplantectomie.

MOTS CLÉS : Transplantation rénale ; Complications vasculaires de la greffe ; Thrombose veineuse du greffon rénal ; Transplantectomie.

\section{JURY :}

Président :

Monsieur le Professeur GUY Laurent

Membres :

Madame le Professeur HENG Anne-Elisabeth,

Monsieur le Professeur SCHMIDT Jeannot,

Monsieur le Docteur GARROUSTE Cyril

Madame le Docteur MILLET Clémentine.

DATE DE LA SOUTENANCE DE THÈSE : 9 avril 2021. 
